\title{
Tides in the High-eccentricity Migration of Hot Jupiters: Triggering Diffusive Growth by Nonlinear Mode Interactions
}

\author{
Hang $\mathrm{Yu}^{1}$ (1), Nevin N. Weinberg ${ }^{2}$ (1), and Phil Arras $^{3}$ (1) \\ ${ }^{1}$ TAPIR, Walter Burke Institute for Theoretical Physics, Mailcode 350-17 California Institute of Technology, Pasadena, CA 91125, USA; hangyu@caltech.edu \\ ${ }^{2}$ Department of Physics, University of Texas at Arlington, Arlington, TX 76019, USA \\ ${ }^{3}$ Department of Astronomy, University of Virginia, P.O. Box 400325, Charlottesville, VA 22904, USA \\ Received 2021 April 11; revised 2021 May 28; accepted 2021 June 9; published 2021 August 12
}

\begin{abstract}
High-eccentricity migration is a possible formation channel for hot Jupiters. However, in order for it to be consistent with the observed population of planets, tides must circularize the orbits in an efficient manner. A potential mechanism for such rapid circularization is the diffusive growth of the tidally driven planetary $f$-mode. Such growth occurs if the $f$-mode phase at pericenter varies chaotically from one pericenter passage to the next. Previous studies focused on the variation of the orbital period due to tidal back-reaction on the orbit as the source of chaos. Here we show that nonlinear mode interactions can also be an important source. Specifically, we show that nonlinear interactions between a parent $f$-mode and daughter $f$ - $/ p$-modes induce an energy-dependent shift in the oscillation frequency of the parent. This frequency shift varies randomly from orbit to orbit because the parents' energy varies. As a result, the parents' phase at pericenter varies randomly, which we find can trigger it to grow diffusively. We show that the phase shift induced by nonlinear mode interactions in fact dominates the shift induced by tidal back-reaction and lowers the one-kick energy threshold for diffusive growth by about a factor of 5 compared to the prediction from the linear theory. For a given pericenter distance, this reduces the semimajor axis needed to trigger diffusive growth from $\gtrsim 2$ au to $\simeq 1$ au. Nonlinear interactions could thus enhance the formation rate of hot Jupiters through the high-eccentricity migration channel and potentially mitigate the discrepancy between the observed and predicted occurrence rates for close-in gas giants as compared to those farther from the star.
\end{abstract}

Unified Astronomy Thesaurus concepts: Exoplanets (498); Hot Jupiters (753); Exoplanet tides (497); Exoplanet migration (2205); Hydrodynamics (1963)

\section{Introduction}

More than $25 \mathrm{yr}$ after the first detection of a hot Jupiter (Mayor \& Queloz 1995), we still do not know their dominant formation channel. Possibilities include in situ formation, gas disk migration, and high-eccentricity tidal migration (see Dawson \& Johnson 2018 for a review). In the latter scenario, the planet is born beyond the snow line at $\gtrsim 1$ au and is driven to high eccentricity through planet-planet scattering (e.g., Rasio \& Ford 1996; Chatterjee et al. 2008) or secular interactions with another planet or star (e.g., Wu \& Murray 2003; Nagasawa et al. 2008; Wu \& Lithwick 2011; Hamers et al. 2017; Teyssandier et al. 2019). Strong tidal interactions during close pericenter passages subsequently damp the eccentricity and shrink the semimajor axis, culminating in a planet that resides in a days-long circular orbit.

An outstanding problem with this formation channel is the lack of very high eccentricity systems $(e>0.9)$ among the observed population of hot Jupiters. In order to sufficiently speed up the circularization and thereby reduce the likelihood of catching a planet in the high- $e$ state, the tidal dissipation must be at least 10 times more efficient than Jupiter (Socrates et al. 2012; Dawson et al. 2015). Such an enhanced efficiency is not necessarily inconceivable, however, as tidal dissipation in hot Jupiter systems can be sensitive to the strength and frequency of the tidal forcing and the structure of the components (see, e.g., Ogilvie \& Lin 2004; Barker 2011; Essick \& Weinberg 2016). One should therefore consider the problem from first principles rather than rely on parameterized extrapolations.

Indeed, $\mathrm{Wu}$ (2018) showed that the diffusive growth of the planet's $l=2 f$-mode during high-eccentricity migration could lead to very rapid orbit circularization (see also Vick \& Lai 2018; Vick et al. 2019). This process was first considered in the hot Jupiter context by Ivanov \& Papaloizou (2004) and has also been considered in a number of other high-eccentricity systems, e.g., in tidal capture binaries (Kochanek 1992; Mardling 1995) and eccentric neutron star binaries (Vick \& Lai 2018). In Wu's calculations, the $f$-mode's phase is randomly perturbed by the back-reaction of the tide on the orbit, causing the mode amplitude to grow diffusively over many pericenter passages. She argued that the $f$-mode will damp nonlinearly when its amplitude reaches unity and the mode breaks near the planet's surface. Within $\sim 10^{4} \mathrm{yr}$, the planet is transported from a few au to $\sim 0.2$ au and its eccentricity is decreased from near unity to $\sim 0.9$ (note, however, that the process does not circularize the orbit all the way to zero eccentricity). Such a rapid circularization is equivalent to a remarkably small tidal quality factor of $\mathrm{QF} \sim 1$, five orders of magnitude smaller than Jupiter's.

Wu (2018) showed that an additional feature of the diffusive growth scenario is that a planet that is secularly perturbed to high eccentricity will dynamically decouple from its perturbers when its pericenter distance reaches $\sim 4$ tidal radii. All migrating Jupiters will therefore park safely outside the zone of tidal disruption, where they are observed today. This may explain why hot Jupiters are formed more efficiently than previous investigations of high-eccentricity tidal migration found: whereas observations show that the observed ratio of hot to cold Jupiters is $\sim 10 \%$, previous theoretical calculations, which did not consider diffusive growth, yielded a ratio of only $\sim 1 \%$ owing to their comparatively high rates of tidal disruption (see Table 2 in Dawson \& Johnson 2018). 
In this paper, we extend the work of Wu (2018) and Vick \& Lai (2018) by considering the effects of weakly nonlinear mode interactions on the $f$-mode's diffusive growth. We show that the random changes in mode phase induced by three-wave nonlinear interactions act in concert with tidal back-reaction on the orbit in order to lower the threshold for diffusive growth. We find that for a given orbital period the diffusive growth can be triggered at a larger pericenter distance (i.e., smaller eccentricity) and hence smaller kick amplitude, as compared to calculations including only linear physics. Here we focus on the triggering and early phases of diffusive growth; an investigation of the long-term evolution will be left to subsequent papers.

The plan of the paper is as follows. Section 2 contains a review of the coupled equations for the mode and orbit including only linear processes. Following Vick \& Lai (2018), an iterative map for the mode amplitude and orbital period is outlined, including the effects of planetary rotation. Nonlinear coupling of the excited $f$-modes with other $f$-and $p$-modes is discussed in Section 3. The nonresonant phase shift and damping rate are derived, and gas giant planet models are used to evaluate the frequencies, damping rates, and coupling coefficients. The nonlinear phase shift and damping are incorporated into the iterative mapping algorithm in Section 4. Results for short-timescale simulations using the maps are presented in Section 5, and conclusions and discussion are presented in Section 6.

\section{Linear Problem}

In this section, we review the linear problem and introduce some of the notation and approximations we will use throughout our study. In Section 2.1, we present the set of equations needed to construct an iterative map of the coupled mode-orbit evolution in linear theory (including the Doppler shifts caused by rotation). Then, in Section 2.2, we justify our approximate treatment of angular momentum transfer and the orbital and spin evolution of the planet.

The eigenmodes form a complete basis for the fluid displacements $\boldsymbol{\xi}(\boldsymbol{x}, t)$, which can be expanded as (Schenk et al. 2002)

$$
\left\{\begin{array}{l}
\boldsymbol{\xi}(\boldsymbol{x}, t)=\sum_{a} q_{a}(t) \boldsymbol{\xi}_{a}(\boldsymbol{x}, t) \\
\dot{\boldsymbol{\xi}}(\boldsymbol{x}, t)=\sum_{a}\left(-i \omega_{a}\right) q_{a}(t) \boldsymbol{\xi}_{a}(\boldsymbol{x}, t)
\end{array}\right.
$$

where $\omega_{a}$ is the eigenfrequency of a mode and $q_{a}$ its amplitude. The sums run over both radial and angular quantum numbers, as well as modes with positive and negative frequencies. We normalize each mode such that

$$
2 \omega_{a}^{2} \int d^{3} x \rho \boldsymbol{\xi}_{a^{\prime}}^{*} \cdot \boldsymbol{\xi}_{a}=E_{0} \delta_{a a^{\prime}}
$$

where $E_{0}=G M^{2} / R, M$ is the mass of the planet, $R$ is its radius, and other quantities have their usual meaning. If we ignore nonlinear effects, the equation of motion of a planetary mode in the frame corotating with the planet is (Schenk et al. 2002)

$$
\dot{q}_{a}+\left(i \omega_{a}+\gamma_{a}\right) q_{a}=i \omega_{a} U_{a}
$$

where $\omega_{a}$ and $\gamma_{a}$ are the eigenfrequency and linear damping rate of the mode, respectively. The amplitude of the tidal force acting on the mode is

$$
U_{a}(t)=W_{l m} Q_{a}\left(\frac{M_{*}}{M}\right)\left[\frac{R}{D(t)}\right]^{l+1} e^{-i m\left[\Phi(t)-\Omega_{\mathrm{s}} t\right]},
$$

where $D$ is the orbital separation, $M_{*}$ is the mass of the host star, $\Phi$ is the orbital phase, $\Omega_{\mathrm{s}}$ is the spin of the planet, the tidal overlap $Q_{a}=\left(M R^{l}\right)^{-1} \int d^{3} r \rho \boldsymbol{\xi}^{*} \cdot \nabla\left(r^{l} Y_{\mathrm{lm}}\right)$, and at leading order $(l=2)$ the nonvanishing $W_{l m}$ coefficients are $W_{2 \pm 2}=\sqrt{3 \pi / 10}$ and $W_{20}=-\sqrt{\pi / 5}$. Note that we include the Doppler shift of frequency due to rotation but ignore corrections to the rotatingframe frequency and eigenfunction for simplicity. Also note that our sign convention is different from that used in $\mathrm{Wu}$ (2018). Specifically, a prograde (retrograde) mode has $m_{a}>0$ $\left(m_{a}<0\right)$ in our definition.

The mode amplitudes couple to the orbital motion through the accelerations $a_{D}$ and $a_{\phi}$ in the equations of motion

$$
\begin{gathered}
\ddot{D}=D \dot{\Phi}^{2}-\frac{G\left(M+M_{*}\right)}{D^{2}}+a_{D}, \\
D \ddot{\Phi}=-2 \dot{D} \dot{\Phi}+a_{\Phi},
\end{gathered}
$$

where to linear order

$$
\begin{gathered}
a_{D}=-\frac{E_{0}}{\mu D} \sum_{a}\left(l_{a}+1\right) \operatorname{Re}\left[q_{a}^{*} U_{a}\right], \\
a_{\Phi}=\frac{E_{0}}{\mu D} \sum_{a} m_{a} \operatorname{Im}\left[q_{a}^{*} U_{a}\right],
\end{gathered}
$$

where $\mu=M M_{*} /\left(M+M_{*}\right)$ is the reduced mass. Throughout our study, we drop the nonlinear tidal back-reaction terms, as their effect on the one-kick amplitude is subdominant.

\subsection{Iterative Map Including Only Linear Effects}

The direct integration of the coupled mode-orbit evolution equations is computationally expensive. To obtain the approximate secular evolution, an iterative mapping procedure has been developed (see, e.g., Vick \& Lai 2018), whose key steps we summarize below (see also similar derivations in Vick et al. 2019).

To do so, we first perform a phase shift to transform Equation (3) from the corotating frame to the inertial frame

$$
\dot{q}_{a}^{\prime}+\left(i \omega_{a}^{\prime}+\gamma_{a}\right) q_{a}^{\prime}=i \omega_{a} U_{a}^{\prime}(t),
$$

where $q_{a}^{\prime}=q_{a} \exp \left(-i m_{a} \Omega_{\mathrm{s}} t\right), \omega_{a}^{\prime}=\omega_{a}+m_{a} \Omega_{\mathrm{s}} t$, and $U_{a}^{\prime}=$ $U_{a} \exp \left(-i m_{a} \Omega_{\mathrm{s}} t\right)$ are, respectively, the mode amplitude, mode frequency, and tidal driving in the inertial frame. The general solution of $q_{a}^{\prime}$ is

$$
q_{a}^{\prime}(t)=e^{-\left(i \omega_{a}^{\prime}+\gamma_{a}\right) t} \int^{t} i \omega_{a} U_{a}^{\prime}(\tau) e^{\left(i \omega_{a}^{\prime}+\gamma_{a}\right) \tau} d \tau
$$

Suppose we know the mode amplitude right before the $k$ th pericenter passage. We can then write the mode amplitude in the $k$ th orbit as (see also Vick et al. 2019)

$$
\begin{gathered}
q_{a, k}^{\prime(0)}=q_{a, k-1}^{\prime(1)}+\Delta q_{a, 1} \\
q_{a, k}^{\prime(1)}=q_{a, k}^{\prime(0)} e^{-\left(i \omega_{a}^{\prime}+\gamma_{a}\right) P_{\mathrm{or}, k},}
\end{gathered}
$$

where the superscripts (0) and (1) indicate that the amplitudes are, respectively, evaluated right after and right before a pericenter passage. The quantity $\Delta q_{a, 1}$ is the one-kick 
amplitude the mode receives at the pericenter. It is given by ${ }^{4}$

$$
\Delta q_{a, 1}=\int i \omega_{a} U_{a}^{\prime}(\tau) e^{\left(i \omega_{a}^{\prime}+\gamma_{a}\right) \tau} d \tau .
$$

In the equation above, the integration is performed over one orbital period. For the rest of the paper, we will only consider highly eccentric orbits with $\left(1-e_{\text {orb }}\right) \ll 1$, where significant tidal interaction happens only near the pericenter. Therefore, the limits of integration in Equation (13) can be dropped as long as they bracket the pericenter passage.

It is convenient to define an orbital integral $K_{l m}$ as ${ }^{5}$ (Press \& Teukolsky 1977)

$$
K_{l m}(\omega)=\frac{\omega_{0} W_{l m}}{2 \pi} \int\left[\frac{D_{\text {peri }}}{D(\tau)}\right]^{l+1} e^{i[\omega \tau-m \Phi(\tau)]} d \tau
$$

where $D_{\text {peri }} \equiv a_{\text {orb }}\left(1-e_{\text {orb }}\right)$ is the pericenter distance, $e_{\text {orb }}$ the eccentricity, and $\omega_{0} \equiv \sqrt{G M / R^{3}}$. If we ignore the perturbations on $D$ and $\Phi$, Lai (1997) provide an analytical expression for $K_{22}$ (i.e., $l=m=2$ ) assuming that the orbit is parabolic,

$$
\begin{aligned}
K_{22}\left(\omega, \Omega_{\text {peri }}\right) & \simeq \frac{2 z^{3 / 2} e^{-2 z / 3}}{\sqrt{15}}\left(\frac{\omega_{0}}{\Omega_{\text {peri }}}\right)\left(1-\frac{\sqrt{\pi}}{4 \sqrt{z}}\right), \\
& \simeq 1.1 \times 10^{-2}\left(\frac{z}{11}\right)^{-5.7}\left(\frac{\omega_{0}}{\Omega_{\text {peri }}}\right),
\end{aligned}
$$

where $z \equiv \sqrt{2} \omega / \Omega_{\text {peri }}$ and $\Omega_{\text {peri }}^{2} \equiv G\left(M+M_{*}\right) / D_{\text {peri }}^{3}$, and in the second line we have expanded the expression around $z=11$ to emphasize the steep decline.

In terms of $K_{l m}$, we can write the one-kick amplitude as

$$
\begin{aligned}
\Delta q_{a, 1}= & i 2 \pi Q_{a} K_{l m}\left(\omega_{a}^{\prime}, \Omega_{\text {peri }}\right) \\
& \times\left(\frac{\omega_{a} \Omega_{\text {peri }}^{2}}{\omega_{0}^{3}}\right)\left(\frac{M_{*}}{M+M_{*}}\right)\left(\frac{R}{D_{\text {peri }}}\right)^{l-2} .
\end{aligned}
$$

The damping term entering $\Delta q_{a, 1}$ can be safely dropped because $\gamma_{a} \ll \Omega_{\text {peri }}$. Note that for a parabolic orbit $K_{l m}$ is a real number and therefore $\Delta q_{a, 1}$ is purely imaginary. Also note that when calculating $K_{l m}$ one should use the mode frequency in the inertial frame $\omega_{a}^{\prime}=\left(\omega_{a}+m \Omega_{\mathrm{s}}\right)$. Combining with the expansion given in Equation (15), one sees immediately that the spin reduces the one-kick amplitude for a prograde mode with $m>0$ in our convention.

To account for the tidal back-reaction on the orbit, we adopt an energy conservation argument instead of explicitly coupling the mode amplitude equation and the tidal accelerations $a_{D}$ and $a_{\Phi}$. Upon receiving a kick at pericenter, the energy stored in a stellar mode changes by (including contributions from the mode and its complex conjugate)

$$
\Delta E_{a, k}=\left[\left|q_{a, k}^{\prime(0)}\right|^{2}-\left|q_{a, k-1}^{\prime(1)}\right|^{2}\right] E_{0} .
$$

\footnotetext{
4 Formally the integration should be performed from right before the $k$ th pericenter passage to right before the next passage. We can nonetheless shift the initial time because $U_{a}^{\prime}(t)=U_{a}^{\prime}\left(t+\sum_{k^{\prime}} P_{\mathrm{orb}, k^{\prime}}\right)$ in the inertial frame if the pericenter distance stays approximately fixed throughout the evolution. This also is the reason the one-kick amplitude can be treated as a constant for different orbital cycles.

5 Note that a tidal field with spherical degree $(l, m)$ linearly couples to a mode with $l_{a}=l$ and $m_{a}=m$ owing to the angular integral in $Q_{a}$.
}

Since the energy stored in the tidal coupling (the term $\left.\propto \operatorname{Re}\left[q_{a}^{*} U_{a}\right]\right)$ is small everywhere except for at the pericenter and the spin rate of the planet should stay approximately fixed (which we will justify shortly), the change in the energy of stellar modes needs to be balanced by the orbital energy,

$$
\Delta E_{\mathrm{orb}, k}=E_{\mathrm{orb}, k}-E_{\mathrm{orb}, k-1}=-\Delta E_{a, k},
$$

where $E_{\mathrm{orb}, k}=-G M M_{*} / 2 a_{\mathrm{orb}, k}$ is the orbital energy at the $k$ th orbit.

A direct consequence is that the change in the orbital energy also alters the orbital period $P_{\text {orb }}=2 \pi / \Omega_{\text {orb }}=$ $2 \pi \sqrt{a_{\mathrm{orb}}^{3} / G\left(M+M_{*}\right)} \propto\left(-E_{\mathrm{orb}}\right)^{-3 / 2}$, as

$$
\frac{\Delta P_{\mathrm{orb}, k}}{P_{\mathrm{orb}, k}}=\frac{3}{2} \frac{\Delta E_{a, k}}{E_{\mathrm{orb}, k}} .
$$

Since the value of $\Delta E_{a, k}$ is different from orbit to orbit, the orbital period varies. This, in turn, leads to a stochastic evolution of the mode's phase per orbital cycle

$$
\Delta \phi_{\mathrm{br}, k}=-\omega_{a} \Delta P_{\mathrm{orb}, k}=-\frac{3}{2} \omega_{a} P_{\mathrm{orb}, k} \frac{\Delta E_{a, k}}{E_{\mathrm{orb}, k}},
$$

where we have used a subscript "br" to stand for the fact that this phase is due to the back-reaction of the tide. As shown in previous studies (Vick \& Lai 2018; Wu 2018), the randomness of the phase shift $\Delta \phi_{\mathrm{br}, k}$ is key to triggering the diffusive growth of a tidally driven mode.

In order to simplify the notation, we will sometime omit the subscript " $k$ " when we do not need the quantity to be evaluated at a specific orbit cycle.

\subsection{Orbital and Spin Angular Momentum}

An energy transfer is typically associated with an angular momentum transfer as well. Nonetheless, since the change in the orbital angular momentum $\Delta L_{\text {orb }} \simeq \Delta E_{\text {orb }} / \Omega_{\text {peri }}$, we have that at high eccentricity (Vick \& Lai 2018)

$$
\left|\frac{\Delta L_{\text {orb }}}{L_{\text {orb }}}\right| \simeq\left|\frac{\Delta E_{a}\left(1-e_{\text {orb }}\right)}{2 \sqrt{2} E_{\text {orb }}}\right| \ll\left|\frac{\Delta E_{a}}{E_{\text {orb }}}\right| .
$$

Therefore, the orbital angular momentum stays as a constant throughout the evolution to a very good approximation. Suppose, for example, the initial orbit is $a_{\text {orb }}=1$ au and $e_{\text {orb }}=0.98$ and it evolves to $a_{\text {orb }}=0.2$ au (with $e_{\text {orb }} \simeq 0.9$ ) owing to tidal dissipation. Whereas the orbital energy changes by a factor of 5 , the orbital angular momentum changes by only $3 \%$ in the process. Furthermore, since the angular momentum is nearly constant and

$$
\begin{aligned}
L_{\mathrm{orb}} & =\mu \sqrt{G\left(M+M_{*}\right) a_{\mathrm{orb}}\left(1-e_{\mathrm{orb}}^{2}\right)} \\
& \simeq \mu \sqrt{2 G\left(M+M_{*}\right) D_{\text {peri }}}
\end{aligned}
$$

at high eccentricity, it follows that the pericenter distance $D_{\text {peri }}$ is also nearly constant throughout the orbital evolution.

Moreover, under the high-eccentricity limit, the linear onekick amplitude $\Delta q_{a, 1}$ depends on the Keplerian elements only through the pericenter distance $D_{\text {peri }}$ (which determines $\Omega_{\text {peri }}$ for fixed $\left.\left(M, M_{*}\right)\right)$. As the pericenter distance stays nearly unchanged, the one-kick amplitude $\Delta q_{a, 1}$ also remains approximately constant. 
So far we have left the spin of the planet $\Omega_{\mathrm{s}}$ as a free parameter. One plausible scenario is that the planet reaches pseudo-synchronization with the orbit via the equilibrium tide, leading to (Hut 1981)

$$
\begin{aligned}
\Omega_{\mathrm{s}} & \simeq \frac{1+\frac{15}{2} e_{\mathrm{orb}}^{2}+\frac{45}{8} e_{\mathrm{orb}}^{4}+\frac{5}{16} e_{\mathrm{orb}}^{6}}{\left(1+3 e_{\mathrm{orb}}^{2}+\frac{3}{8} e_{\mathrm{orb}}^{4}\right)\left(1-e_{\mathrm{orb}}^{2}\right)^{3 / 2}} \Omega_{\mathrm{orb}}, \\
& \simeq 1.17 \Omega_{\mathrm{peri}} \quad\left(e_{\mathrm{orb}} \rightarrow 1\right),
\end{aligned}
$$

where the second line applies in the high-eccentricity limit. A constant pericenter distance (hence constant $\Omega_{\text {peri }}$ ) would then imply that the spin frequency as set by the pseudosynchronization condition also stays approximately constant. We note that including a pseudo-synchronous rotation of the planet will increase the prograde $f$-mode frequency in the inertial frame, which will tend to decrease the one-kick amplitude and slow the orbital evolution as compared to the nonrotating planet case. Nevertheless, the one-kick amplitude of the prograde mode is still two orders of magnitude greater than the $m_{a}=0$ mode and even more for the retrograde mode. Therefore, we will only consider the prograde mode in the subsequent discussion.

To summarize our procedure, we discard the evolution of the angular momenta and, self-consistently, treat $\Omega_{\mathrm{s}}$ and $D_{\text {peri }}$ as approximate constants during the circularization process (at least for the initial phase when $1-e_{\text {orb }} \ll 1$ is well satisfied). The evolution trajectories will thus reduce to the ones studied by Vick \& Lai (2018) and Wu (2018) as long as one uses the mode frequency in the inertial frame $\omega_{a}^{\prime}=\omega_{a}+m_{a} \Omega_{\mathrm{s}}$ and neglects the nonlinear effects described in the next section.

\section{Nonlinear Problem}

We now consider how weakly nonlinear effects modify the problem. At lowest nonlinear order, the amplitude equation of a mode $a$ is (Weinberg et al. 2012)

$$
\dot{q}_{a}+\left(i \omega_{a}+\gamma_{a}\right) q_{a}=i \omega_{a}\left[U_{a}+\sum_{b} U_{a b}^{*} q_{b}^{*}+\sum_{b c} \kappa_{a b c} q_{b}^{*} q_{c}^{*}\right]
$$

where $U_{a b}$ is the nonlinear tide, $\kappa_{a b c}$ is the three-mode coupling coefficient, and asterisks denote complex conjugation. There is a significant cancellation between the nonlinear tide and three-mode coupling to the equilibrium tide such that $U_{a b}+2 \sum_{c} \kappa_{a b c} U_{c} \simeq 0$ (Weinberg et al. 2012). By treating the cancellation as perfect, we have

$$
\begin{aligned}
& \dot{q}_{a}+\left(i \omega_{a}+\gamma_{a}\right) q_{a}=i \omega_{a} U_{a} \\
& \quad+i \omega_{a} \sum_{b c} \kappa_{a b c}\left(q_{b}^{*} q_{c}^{*}-q_{b}^{*} U_{c}^{*}-q_{c}^{*} U_{b}^{*}\right) .
\end{aligned}
$$

We will solve this equation (or approximate its solution) in order to determine how nonlinear mode interactions influence the diffusive growth of the $f$-mode and thereby a planet's higheccentricity tidal migration.

Since the daughters' direct, linear coupling to the tide is small, we expect the most significant nonlinear effect to be the modification of the parent mode's free evolution away from pericenter (when $U_{a} \simeq 0$ ). Specifically, we show in Section 3.1 that the nonlinear mode couplings can be viewed as energydependent shifts of the parent's eigenfrequency and damping rate. We then derive the time-dependent evolution of such a nonlinear oscillator in Section 3.2. Lastly, we examine the nonlinear effects in a typical Jupiter model in Section 3.3.

\subsection{Nonlinear Frequency Shift and Effective Damping Rate}

Previous studies have shown that at linear order the $l_{a}=m_{a}=2 f$-mode with $\omega_{a}>0$ has the greatest energy and that it dominates the orbital evolution (see, e.g., Wu 2018). We will thus focus on a single parent mode (mode $a$ ) with $l_{a}=m_{a}=2$ and $\omega_{a}>0$ (and its complex conjugate to get real, physical quantities). We consider the nonlinear effects due to this parent mode coupling to itself and a daughter mode (which can be another $f$-mode or a $p$-mode), which results in the inhomogeneous driving of the daughter. As we consider fully convective Jupiter models, there are no low-frequency $g$-modes that can parametrically couple to the parent (Weinberg et al. 2012). Therefore, the nonresonant nonlinearity considered in this work should be distinguished from the parametric instabilities considered in, e.g., Essick \& Weinberg (2016) for solar-type stars and Yu et al. (2020) for white dwarfs. ${ }^{6}$

Once we know the parent mode's angular pattern $\left(l_{a}, m_{a}\right)$, we can further utilize the three-mode angular selection rules and divide up the nonlinear couplings into two categories, which we will refer to as $a a b$ and $a a^{*} c$, respectively.

In the $a a b$ case, the driving is formed by mode $a$ coupling to itself. By the angular section rule, only daughter modes with $l_{b}=-m_{b}=4$ can couple to this driving. We will refer to such a daughter as mode $b$ and note that it is forced at a frequency $-2 \omega_{a}$.

By contrast, in the $a a^{*} c$ case, mode $a$ couples to its complex conjugate $a^{*}$ and drives a daughter mode (mode $c$ ) with $m_{c}=0$ and $l_{c}=0,2,4$. In this case, mode $c$ experiences a forcing at zero frequency. ${ }^{7}$

To make the abstract problem more transparent, we write out the explicit three-mode coupling equations for both cases. For simplicity, we start by considering only a single mode $b$ and a single mode $c$. We will perform a summation over modes in the end to obtain the general solution. We will also drop the couplings involving more than one daughter mode for analytical simplicity; all the allowed couplings are included in the numerical calculations when we validate our analytical approximations. We then have

$$
\begin{gathered}
\dot{q}_{a}+\left(i \omega_{a}+\gamma_{a}\right) q_{a}=2 i \omega_{a} \kappa_{b} q_{b}^{*} q_{a}^{*}+2 i \omega_{a} \kappa_{c} q_{c}^{*} q_{a}, \\
\dot{q}_{b}+\left(i \omega_{b}+\gamma_{b}\right) q_{b}=i \omega_{b} \kappa_{b} q_{a}^{*} q_{a}^{*} \\
\dot{q}_{c}+\left(i \omega_{c}+\gamma_{c}\right) q_{c}=2 i \omega_{c} \kappa_{c} q_{a}^{*} q_{a} .
\end{gathered}
$$

Note that the above set of equations describes the evolution away from pericenter, after the parent has received its most recent kick, since here we are interested in following the

\footnotetext{
6 The planet may support gravity waves ( $g$-modes) above the radiative - convective boundary in the surface (Jermyn et al. 2017). Alternatively, if a Jupiter also has a diffusive core similar to Saturn (Mankovich \& Fuller 2021), core $g$-modes might also exist. However, the potential effects of the planetary $g$-modes are left for future studies.

Care must be taken when such DC forcing is encountered in the nonlinear problem in order to ensure that the forcing represents the physical transfer of energy and angular momentum between distinct oscillation modes, and not just a constant nonlinear modification of the linear mode frequency and eigenfunction. In the present situation, there is additional time dependence due to the mode amplitudes, which change from one orbit to the next, and such forcing in turn leads to further time-dependent changes in the modes' amplitude and phase.
} 
parent's free (i.e., unforced) evolution leading up to the next pericenter passage. Consequently, we do not include any tidal forcing terms.

To seek the leading-order nonlinear correction, we solve Equations (26)-(28) in a perturbative manner. Away from pericenter and without nonlinear couplings, we have $q_{a} \sim \exp \left(-i \omega_{a} t\right)$. Using this as the driving term, the steadystate solutions of the daughters are ${ }^{8}$

$$
\begin{gathered}
q_{b}=\frac{\omega_{b}\left(2 \omega_{a}+\omega_{b}\right)+i \omega_{b} \gamma_{b}}{\left(2 \omega_{a}+\omega_{b}\right)^{2}+\gamma_{b}^{2}} \kappa_{b} q_{a}^{*} q_{a}^{*}, \\
q_{c}=\frac{2 \omega_{c}^{2}+2 i \omega_{c} \gamma_{c}}{\omega_{c}^{2}+\gamma_{c}^{2}} 2 \kappa_{c} q_{a}^{*} q_{a} .
\end{gathered}
$$

Plugging the daughter modes above back into Equation (26), we obtain

$$
\dot{q}_{a}+\left[i\left(\omega_{a}+\delta \omega_{a}\right)+\left(\gamma_{a}+\delta \gamma_{a}\right)\right] q_{a}=0,
$$

where ${ }^{9}$

$$
\begin{aligned}
& \delta \omega_{a}=-\omega_{a}\left[\frac{2 \omega_{b}\left(2 \omega_{a}+\omega_{b}\right)}{\left(2 \omega_{a}+\omega_{b}\right)^{2}+\gamma_{b}^{2}} \kappa_{b}^{2}+\frac{4 \omega_{c}^{2}}{\omega_{c}^{2}+\gamma_{c}^{2}} \kappa_{c}^{2}\right] \tilde{E}_{a} \\
& \delta \gamma_{a}=-\omega_{a}\left[\frac{2 \omega_{b} \gamma_{b}}{\left(2 \omega_{a}+\omega_{b}\right)^{2}+\gamma_{b}^{2}} \kappa_{b}^{2}+\frac{4 \omega_{c} \gamma_{c}}{\omega_{c}^{2}+\gamma_{c}^{2}} \kappa_{c}^{2}\right] \tilde{E}_{a},
\end{aligned}
$$

and $\tilde{E}_{a} \equiv q_{a}^{*} q_{a}$ is the dimensionless energy of mode $a .{ }^{10}$ The physical mode energy including the contribution from both $a$ and its complex conjugate $a^{*}$ is $E_{a}=\tilde{E}_{a} E_{0}$ in our normalization, where $E_{0}=G M^{2} / R$ is the natural energy of the planet.

We thus see that the leading-order nonlinear correction corresponds to a shift in the eigenfrequency (conservative part) of the parent mode and an excess damping term (dissipative part), both of which depend linearly on the energy of the parent mode (see also Landau \& Lifshitz 1976; Kumar et al. 1994; Kumar \& Goodman 1996). We can therefore define

$$
\begin{aligned}
& \delta \omega_{a}\left(\tilde{E}_{a}\right)=\Omega \tilde{E}_{a}, \\
& \delta \gamma_{a}\left(\tilde{E}_{a}\right)=\Gamma \tilde{E}_{a},
\end{aligned}
$$

where, after putting back the summation over all the daughters that couple to mode $a$, we have

$$
\begin{gathered}
\Omega=-\omega_{a}\left[\sum_{b} \frac{2 \omega_{b}\left(2 \omega_{a}+\omega_{b}\right)}{\left(2 \omega_{a}+\omega_{b}\right)^{2}+\gamma_{b}^{2}} \kappa_{b}^{2}+\sum_{c} \frac{4 \omega_{c}^{2}}{\omega_{c}^{2}+\gamma_{c}^{2}} \kappa_{c}^{2}\right], \\
\Gamma=\sum_{b} \frac{-2 \omega_{a} \omega_{b}}{\left(2 \omega_{a}+\omega_{b}\right)^{2}+\gamma_{b}^{2}} \gamma_{b} \kappa_{b}^{2} .
\end{gathered}
$$

Note that mode $c$ does not contribute to the nonlinear damping $\Gamma$. Mathematically, this can be understood by noticing that for every mode $c$ with $\omega_{c}$ there exists a mode $c^{*}$ with $-\omega_{c}$ (i.e., the complex conjugate of $c$; they both have $m_{c}=m_{c^{*}}=0$ )

\footnotetext{
8 These solutions involve some approximations that we discuss in Section 4 and the Appendix.

9 We use " $\Delta$ " to indicate the difference between adjacent orbital cycles and " $\delta$ " for the nonlinear deviation relative to the linear case.

10 For the rest of the paper, we will use the "tilde" symbol to represent dimensionless energies (i.e., energies normalized by the natural energy of the planet, $\left.E_{0}=G M^{2} / R\right)$.
}

that has the exact opposite contribution to $\Gamma$. Therefore, after summing over the $\pm \omega_{c}$ pair, the nonlinear dissipation due to mode $c$ cancels exactly. Physically, we can view mode $c$ as a nonlinear modification of the planet's structure, which changes the frequency at which the parent wave propagates (see footnote 7). Nonetheless, mode $c$ is not a wave itself (as it is nonoscillatory), and therefore it does not contribute to the energy dissipation.

By contrast, mode $b$ (corresponding to a nonlinearly excited wave oscillating at $2 \omega_{a}$ ) enhances the dissipation, as one would expect physically. After the summation, $\delta \gamma_{a}$ is always positive because a mode $b$ with negative (positive) frequency would contribute a positive (negative) dissipation rate, and $\left(2 \omega_{a}+\left|\omega_{b}\right|\right)>\left(2 \omega_{a}-\left|\omega_{b}\right|\right)$ as the parent mode has $\omega_{a}>0$. Consequently, we obtain a net increase in the damping after summing over each $\pm \omega_{b}$ pair.

Now turn to the nonlinear frequency shift $\Omega$. Its sign is not definite. While most of the modes ${ }^{11}$ act to reduce the parent mode's frequency, a mode $b$ with $\omega_{b}<0$ and $\left(2 \omega_{a}+\omega_{b}\right)>0$ will increase the parent mode's frequency. In practice, we find that only the $l_{b}=-m_{b}=4 f$-mode satisfies the condition $\omega_{b}\left(2 \omega_{a}+\omega_{b}\right)<0$; this mode can in fact be resonant with the parent, although we find that it is only a mild resonance since the mode spectrum is sparse for low-order (in both $n$ and $l$ ) modes. Therefore, in general we would expect $\Omega<0$. However, in principle it could be positive if there is a rare strong resonance such that $\left(2 \omega_{a}+\omega_{b}\right) \simeq 0^{+} .{ }^{12}$

\subsection{Evolution of the Nonlinear Oscillator}

In the previous section, we showed that nonlinear mode interactions perturb the frequency of the $f$-mode by $\delta \omega_{a}\left(\tilde{E}_{a}\right)$ and its damping rate by $\delta \gamma_{a}\left(\tilde{E}_{a}\right)$. These cause a dephasing of the $f$-mode $\delta \phi_{\mathrm{nl}}=-\int \delta \omega_{a} d t$ in excess of the back-reaction of the $f$-mode on the tide considered in previous studies of diffusive growth (e.g., Wu 2018). Furthermore, this change in phase varies from orbit to orbit owing to the changes in the parent energy. Nonlinear effects can therefore contribute to, and even trigger (as we will show), diffusive growth. Since $\delta \omega_{a}$ depends on the energy of the mode $\tilde{E}_{a}$, in order to determine $\delta \phi_{\mathrm{nl}}$ as a function of time, we need to determine how $\tilde{E}_{a}$ evolves. Note that here we focus on the evolution when the planet is far from pericenter, i.e., of the free oscillator. The goal of this section is therefore to determine $\tilde{E}_{a}(t)$ over an orbit and from it calculate the nonlinear contributions to the dephasing $\delta \phi_{\mathrm{nl}}$. The construction of an iterative mapping from orbit to orbit similar to that of the linear studies will be discussed later in Section 4.

The energy evolution is given by ${ }^{13}$

$$
\dot{\tilde{E}}_{a}+2\left[\gamma_{a}+\delta \gamma_{a}\left(\tilde{E}_{a}\right)\right] \tilde{E}_{a}=0 .
$$

\footnotetext{
${ }^{11}$ This includes all of the mode $c$ type modes (with $m_{c}=0$ ), all the positivefrequency mode $b$ type modes (with $m_{b}=-4$ and $\omega_{b}>0$ ), and the negativefrequency ones with $\left|\omega_{b}\right|>2 \omega_{a}$.

12 Note that the detuning $\left(2 \omega_{a}+\omega_{b}\right)$ is not affected by the choice of reference frame (inertial or corotating) as one would expect physically. This is guaranteed by the angular selection rule $2 m_{a}+m_{b}=0$, which exactly cancels the Doppler shifts.

${ }^{13}$ Here we have implicitly assumed that the parent mode's energy dominates the total energy stored in the stellar oscillations, which is a reasonable approximation in the case we consider here.
} 
Table 1

Properties of the Jupiter Model Considered in Our Study

\begin{tabular}{lcccccc}
\hline \hline$R$ & $E_{0,43}$ & $\omega_{0,-4}$ & $\omega_{\text {prnt }}$ & $Q_{\text {prnt }}$ & $\Omega$ & $\Gamma_{-10}$ \\
\hline $1.1 R_{\mathrm{J}}$ & 3.1 & 5.1 & $1.1 \omega_{0}$ & 0.43 & $-29 \omega_{\text {prnt }}$ & $8.1 \omega_{\text {prnt }}$ \\
\hline
\end{tabular}

Note. We write $E_{0}=G M^{2} / R=E_{0,43} \times 10^{43} \mathrm{erg}, \omega_{0}=\sqrt{G M / R^{3}}=\omega_{0,-4} \times$ $10^{-4} \mathrm{rad} \mathrm{s}^{-1}$, and $\Gamma=\Gamma_{-10} \times 10^{-10}$. We use the subscript "prnt" to denote the parent mode (the prograde $f$-mode with $l_{a}=2$ ) that directly couples to the tide.

If we substitute in Equation (35) for $\delta \gamma_{a}\left(\tilde{E}_{a}\right)$, then the above equation can be solved easily as

$$
\begin{aligned}
\tilde{E}_{a}(t) & =\frac{\gamma_{a} \tilde{E}_{a}^{(0)}}{-\Gamma \tilde{E}_{a}^{(0)}+\left[\gamma_{a}+\Gamma \tilde{E}_{a}^{(0)}\right] e^{2 \gamma_{a} t}} \\
& \simeq \frac{\tilde{E}_{a}^{(0)}}{1+2\left[\gamma_{a}+\Gamma \tilde{E}_{a}^{(0)}\right] t},
\end{aligned}
$$

where $\tilde{E}_{a}^{(0)}$ is the initial mode energy and in the second line we expand $\exp \left[2 \gamma_{a} t\right] \simeq\left(1+2 \gamma_{a} t\right)$. This is a good approximation because the linear damping of the parent mode is typically small and over the course of a $\sim 1$ yr orbit the condition $\gamma_{a} t \ll 1$ is very well satisfied (for the Jupiter model considered here, we have $1 / \gamma_{a} \simeq 10^{9}$ yr or a quality factor $\mathrm{QF} \sim \omega_{a} / \gamma_{a} \simeq 10^{13}$; see also Goldreich \& Nicholson 1977); it is well satisfied even if one uses the empirically determined value of $Q F \simeq 10^{5}$ based on the Jupiter-Io system (see, e.g., Lainey et al. 2009).

The total accumulated phase can be written as $\phi_{a}(t)=$ $-\int\left[\omega_{a}^{\prime}+\delta \omega_{a}\left(\tilde{E}_{a}\right)\right] d t$. Of particular interest is the excess dephasing due to nonlinear interactions

$$
\delta \phi_{\mathrm{nl}}=-\int \delta \omega_{a}\left[\tilde{E}_{a}(t)\right] d t=-\int \frac{\delta \omega_{a}\left(\tilde{E}_{a}\right)}{d \tilde{E}_{a} / d t} d \tilde{E}_{a},
$$

where we use the subscript "nl" to indicate the excess phase due to nonlinear effects (in contrast to tidal back-reactions denoted by a subscript "br"), and in the second equality we change variables from time $t$ to energy $\tilde{E}_{a}$. If we use Equation (34) for $\delta \omega_{a}$ and Equation (38) for $d \tilde{E}_{a} / d t$, then the nonlinear dephasing is

$$
\delta \phi_{\mathrm{nl}}\left(\tilde{E}_{a}\right)=\frac{\Omega}{2 \Gamma} \ln \left[\frac{\gamma_{a}+\Gamma \tilde{E}_{a}}{\gamma_{a}+\Gamma \tilde{E}^{(0)}}\right] .
$$

We can also write the (leading-order) dephasing as a function of time by plugging in Equation (39). In the limit that the parent mode's dissipation is small $\left(\gamma_{a} t \rightarrow 0\right)$, we can cast the dephasing in an intuitive form as

$$
\begin{aligned}
\delta \phi_{\mathrm{nl}}(t) \simeq & -\frac{\Omega}{2 \Gamma} \ln \left[1+2 \Gamma \tilde{E}_{a}^{(0)} t\right], \\
& \simeq-\Omega \tilde{E}_{a}^{(0)} t,
\end{aligned}
$$

where in the second equality we further assumed $2 \Gamma_{a} \tilde{E}_{a}^{(0)} t \ll 1$. For $\tilde{E}_{a}^{(0)} \simeq 10^{-3}$ and $t \simeq 1 \mathrm{yr}$, this condition is satisfied if $\Gamma / \omega_{a}<10^{-5}$. As we will see shortly in the following section and Table 1, Equation (43) is well satisfied for the Jupiter model we consider in this work, as it has a weak damping. Nonetheless, for Jupiters with greater radii, the damping rate can be significantly higher (Arras \& Socrates 2009) and
Equation (42) should be used instead. Obviously, Equation (43) also applies for conservative systems.

\subsection{Values of $\Omega$ and $\Gamma$ for a Jupiter Model}

From the discussion above, we see that the leading-order nonlinear corrections to the parent mode correspond to shifts in both the eigenfrequency and the damping rate that are linearly proportional to the mode energy $\tilde{E}_{a}$ (Equations (34) and (35)). The nonlinear dynamics can thus be characterized by the two coefficients $\Omega$ and $\Gamma$ (both having the dimension of frequency in our definition). These coefficients further depend on the parent mode's eigenfrequency (with $\Omega, \Gamma \propto \omega_{a}$ ) and the properties of the daughters. We now describe values for $\Omega$ and $\Gamma$ for a Jupiter model with $M=M_{\mathrm{J}}$ and $R=1.1 R_{\mathrm{J}}$.

Using MESA (version 10398; Paxton et al. 2011, 2013, 2015, 2018, 2019), we construct a planetary model with a total mass equal to Jupiter's $M=M_{\mathrm{J}}$ and a core mass of $5 M_{\oplus}$ (though the results should be insensitive to the core, as the eigenfunctions of both $f$ - and $p$-modes are largest near the surface). We then let the model contract until it reaches a desired radius, which we choose to be $R=1.1 R_{\mathrm{J}}$. Irradiation is turned on in this contraction phase with a fixed flux of $6.8 \times 10^{6} \mathrm{erg} \mathrm{cm}^{-2} \mathrm{~s}^{-1}$, which corresponds to the average flux the planet receives assuming an orbit with $\left(a_{\text {orb }}, e_{\text {orb }}\right)=(1 \mathrm{au}$, 0.98 ) and a solar-type host star (the equilibrium temperature is $\simeq 420 \mathrm{~K}$ ). After the construction of the background model, we find the (adiabatic) eigenmodes (including both the parent $f$ mode and the leading-order daughter $f$ - and $p$-modes) using GYRE (Townsend \& Teitler 2013; Townsend et al. 2018). The three-mode coupling coefficient is calculated using the expression in Weinberg et al. (2012). We account for the damping of each mode due to turbulent convection using the approach described in Appendix B3 of Burkart et al. (2013).

The key parameters of the Jupiter model are summarized in Table 1. Of particular interest are the values of $\Omega$ and $\Gamma$. We find that $\Omega$ is typically negative and of order $\left|\Omega / \omega_{\text {prnt }}\right| \sim 30$, where here we will use subscript " $a$ " to stand for a generic mode and "prnt" to indicate the parent mode specifically. The value of $\Gamma$ will always be positive, as argued in Section 3.1.

To illustrate the values of the parameters that enter the calculation of $\Omega$ and $\Gamma$, we present the eigenfrequency, threemode-coupling coefficients, and the linear damping rate of each daughter in Figure 1. In addition, we show in Figure 2 each daughter's contribution to $\Omega$ (top panel) and $\Gamma$ (bottom panel). Note that only the negative-frequency, $\left(l_{a}, m_{a}, n_{a}\right)=(4,-4,0)$ $f$-mode contributes a positive value to $\Omega$. Although it has the greatest single-mode contribution, as it is the most resonant daughter with respect to the parent's driving, the resonance is not particularly strong. ${ }^{14}$ Instead, upon summing over all couplings, the value of $\Omega$ is dominated by the coupling to the many $m_{a}=0$ modes ("mode c" in Section 3.1), as well as the off-resonant $m_{a}=-4$ modes ("mode b" with $\left|\omega_{b}\right|>2\left|\omega_{a}\right|$ ), and is therefore negative.

By contrast, only the $m_{a}=-4$ daughters (oscillating at $\left.-2 \omega_{\text {prnt }}\right)$ contribute to the damping, and $\Gamma$ is always positive (see both Section 3.1). For the model we consider here, $\Gamma$ has a particularly small value of $\Gamma / \omega_{\text {prnt }} \sim 10^{-9}$. For Jupiters with $R>1.1 R_{\mathrm{J}}, \Gamma$ may be significantly larger. In part, this is because

\footnotetext{
14 The Jupiter model we consider here has $\left|\omega_{b} /\left(2 \omega_{a}+\omega_{b}\right)\right| \simeq 27$. As a comparison, for a constant-density, incompressible sphere, the $f$-mode eigenfrequency follows $\omega_{a} \propto \sqrt{2 l_{a}\left(l_{a}-1\right) /\left(2 l_{a}+1\right)}$, which leads to a similarly large number $\left|\omega_{b} /\left(2 \omega_{a}+\omega_{b}\right)\right| \simeq 10$.
} 


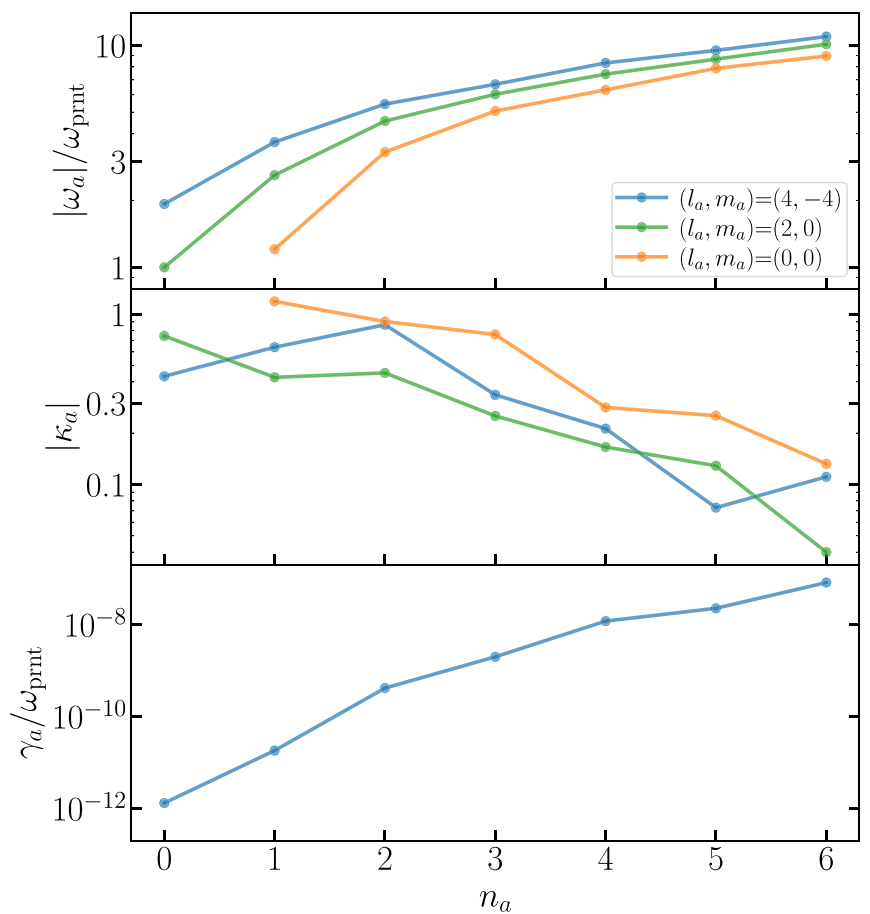

Figure 1. Eigenfrequency (top), three-mode coupling coefficient (middle), and linear damping rate (bottom) of each daughter mode. Here the subscript $a$ in the labels stands for a generic mode, and we specifically label the parent mode's eigenfrequency as $\omega_{\text {prnt }}$. For a daughter with $\left|m_{a}\right|=4$, the coupling we consider is specifically due to parent-parent-daughter, and for an $m_{a}=0$ daughter, it is due to parent-parent ${ }^{*}$-daughter. We only show the damping rate for the $\left|m_{a}\right|=4$ modes, as only they contribute to the energy dissipation.

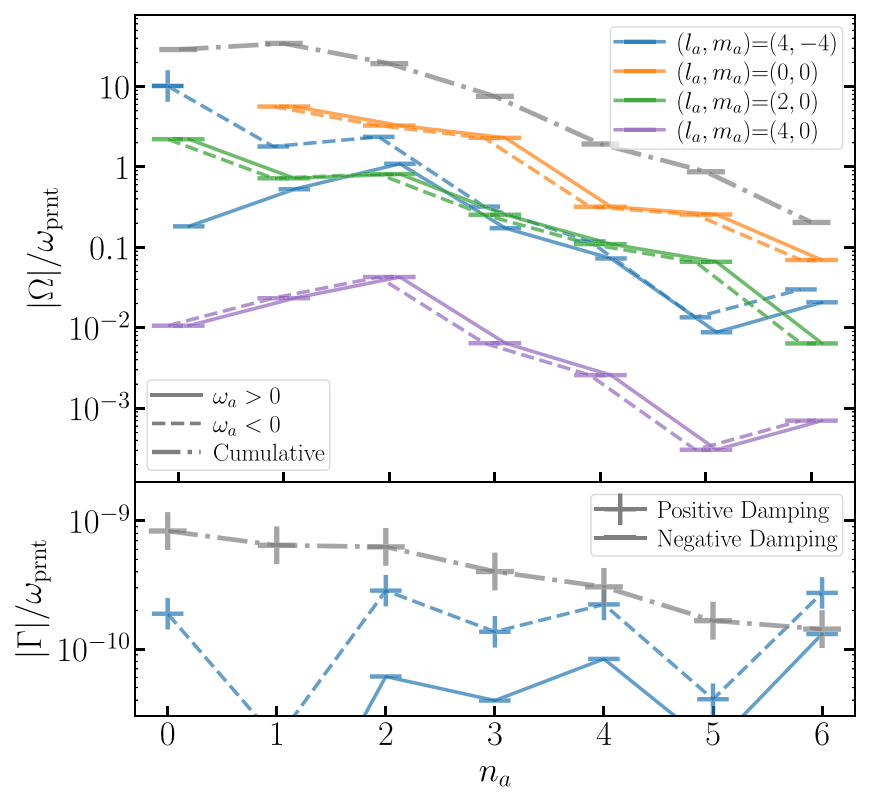

Figure 2. Each daughter mode's contribution to the sums that comprise the nonlinear frequency shift $\Omega$ (top panel) and nonlinear damping $\Gamma$ (bottom panel). The $x$-axis is the radial order of the daughter, with $n_{a}=0$ for $f$-modes and $n_{a}>0$ for $p$-modes. We use different colors to label daughter modes with different $(l, m)$ and solid/dashed lines to label positive/negative-frequency modes. For clarity, we shifted the radial order by $+0.1(-0.1)$ for modes with positive (negative) frequencies. If a daughter mode contributes a positive value to $\Omega(\Gamma)$, a plus sign is used; otherwise, we use a minus sign to show the negative contribution. In the gray, dashed-dotted traces, we also show the cumulative values of $\Omega$ and $\Gamma$ obtained by summing over daughter modes with all the possible $\left(l_{a}, m_{a}, \omega_{a}\right)$ and with radial order $n_{a}^{\prime} \geqslant n_{a}$. The leftmost gray markers thus correspond to the values of $\Omega$ and $\Gamma$. the damping rate due to turbulent convection increases sharply with increasing $R$ (Arras \& Socrates 2009). In addition, we find that irradiation causes a thin radiative zone to form near the surface where the daughter $p$-modes' shears peak. This further reduces the dissipation due to turbulent convection compared to the case without irradiation.

From this point onward, we will use values shown in Table 1 as the default parameters for the planetary model. A primary goal of this paper is to develop the theoretical framework for diffusive tidal evolution including nonlinear mode interactions. A more comprehensive survey on how the tidal evolution trajectories depend on different values of $(\Omega, \Gamma)$ and how $(\Omega, \Gamma)$ further vary with respect to $(M, R)$ is deferred to future work.

\section{Iterative Map Including Nonlinear Effects}

We now have the ingredients to perform an iterative map similar to the one used by Vick \& Lai (2018) but now including nonlinear effects. Since we consider here a single parent mode $a$, and the daughters' effects are collectively absorbed into $\Omega$ and $\Gamma$, we will drop the subscript $a$ in mode amplitude and energy from this point onward.

Suppose the parent mode has an amplitude $q_{k-1}^{\prime(1)}$ in the inertial frame right before the $k$ th pericenter passage. Its amplitude right after the $k$ th passage, $q_{k}^{\prime(0)}$, is given by Equation (11), just as in the linear case (but see the discussion below of the potential impact of nonlinear effects on the parent's kick). Given $q_{k}^{\prime(0)}$, the orbital energy and period of the $k$ th cycle are given by Equations (18) and (19), respectively.

The next step of the mapping is to relate $q_{k}^{\prime(0)}$ to $q_{k}^{\prime(1)}$, the amplitude right before the $(k+1)$ th passage. While the model we consider in Section 3.3 has a particularly weak dissipation, to obtain the accumulated phase over the course of the $k$ th orbit one needs to account for the gradual decay of the parent's energy due to linear and nonlinear damping. This can be achieved by first obtaining the energy $\tilde{E}_{k}^{(1)}=\left|q_{k}^{\prime(1)}\right|^{2}$ using the second line of Equation (39) with $\tilde{E}_{a}^{(0)}=\tilde{E}_{k}^{(0)}$ and $t=P_{\text {orb }, k}$. The evolution phase right before the $(k+1)$ th passage is then

$$
\phi_{k}=-\omega_{a}^{\prime} P_{\mathrm{orb}, k}+\delta \phi_{\mathrm{nl}, k}
$$

Here $\delta \phi_{\mathrm{nl}, k}$ is the excess phase due to the nonlinear frequency shift (the frequency shift due to linear damping is negligible since $\gamma_{a} \ll \omega_{a}^{\prime}$ ), which can be calculated using Equation (43) with $\tilde{E}_{a}^{(0)}=\tilde{E}_{k}^{(0)}$ and $\tilde{E}_{a}=\tilde{E}_{k}^{(1)} \cdot 15$

Before we proceed, it is important to point out a few caveats to this approach. First of all, the expressions we derive in this work are only the leading-order nonlinear corrections. They are accurate only when the parent mode's energy satisfies $\tilde{E}_{k} \lesssim 10^{-3}$. Therefore, in this work our focus will be on the initial triggering of the diffusive tide by the nonlinear mode coupling, particularly the nonlinear phase shift $\propto \Omega$. The evolution timescale we consider here is thus typically a few hundreds of years or less, when the parent mode is still building up its energy. We defer the examination of tidal evolution over $\sim 10 \mathrm{kyr}$ to follow-up studies in this series, as such a study would require both modifications to our leading-order

\footnotetext{
15 If a system is strictly conservative, then we have $\tilde{E}_{k}^{(1)}=\tilde{E}_{k}^{(0)}$, and one can use Equation (43) with $t=P_{\text {orb, } \mathrm{k}}$ to obtain the phase.
} 
expressions and energy dissipation mechanisms due to both weakly nonlinear damping $\propto \Gamma$ and strongly nonlinear wavebreaking as considered in $\mathrm{Wu}$ (2018). ${ }^{16}$

Second, we assumed that the daughters' amplitudes are given by their instantaneous steady-state values, Equations (29) and (30). We show in the Appendix that this may not be strictly true if a daughter mode $b$ (with $\left|m_{b}\right|=4$ ) has $\left|2 \omega_{a}+\omega_{b}\right| \lesssim \Omega_{\text {peri. }}$. Specifically, there should be an additional contribution to the daughter's amplitude that depends on the past history of the mode network. Nonetheless, we drop such corrections for simplicity in the current study. Our numerical experiments suggest that this term becomes potentially important only after a few thousand orbital cycles and therefore should not affect the initial triggering of the diffusive process we consider in this work.

Lastly, we have assumed that the "kick" at each pericenter passage is always given by the linear calculation. In reality, the kick $\Delta q_{1}$ depends on the parent's eigenfrequency (through $K_{l m}$; Equation (15)), which changes nonlinearly. Consequently, $\Delta q_{1}$ should also be modified by the nonlinear frequency shift. However, for $\tilde{E}_{k} \lesssim 10^{-3}$, the fractional decrease of the parent's eigenfrequency $\Omega E \tilde{E}_{k} / \omega_{a}$ is only a few percent. ${ }^{17}$ The change in the one-kick amplitude is thus less than $20 \%$ according to Equation (15). Its effect can be more significant as the parent mode's energy further builds up, however; we plan to examine this in follow-up studies.

\section{Triggering Diffusive Growth}

\subsection{Relative Importance of Linear and Nonlinear Effects for Triggering Diffusive Growth}

The main question we want to investigate in this paper is, how do nonlinear mode interactions affect the threshold for triggering the diffusive growth of the $f$-mode? In order to trigger diffusive growth, the phase evolution of the mode must vary randomly from orbit to orbit by an amount (Vick \& Lai 2018; Wu 2018)

$$
\left|\Delta \phi_{k}\right|=\left|\phi_{k}-\phi_{k-1}\right|>\mathcal{O}(1) \mathrm{rad}
$$

where $\phi_{k}$ is given by Equation (44). In linear theory, this is achieved through the tidal back-reaction on the orbit. At each passage, a random amount of energy $\Delta \tilde{E}_{k}$ is removed from the orbit, which changes the orbital period by $\Delta P_{\text {orb }, k}$ (Equation (19)) and consequently the phase by $\Delta \phi_{\mathrm{br}, k}=-\omega_{a}^{\prime} \Delta P_{\mathrm{orb}, k}$, where the subscript "br" stands for "back-reaction." However, linear theory neglects the fact that the energy $\Delta \tilde{E}_{k}$ gained by the planet's $f$-mode also changes its eigenfrequency by $\Delta \omega_{a}\left(\Delta \tilde{E}_{k}\right)=$ $\delta \omega_{a}\left(\tilde{E}_{k}\right)-\delta \omega_{a}\left(\tilde{E}_{k-1}\right) \simeq \Omega \Delta \tilde{E}_{k}$. This frequency shift induces an additional random phase variation (relative to the previous orbit) $\Delta \phi_{\mathrm{nl}, k} \simeq-\left(\Omega \Delta \tilde{E}_{k}\right) P_{\mathrm{orb}, k}$. The nonlinear frequency shift therefore provides another way of triggering the $f$-mode's diffusive growth.

Quantitatively, Vick \& Lai (2018) found that it is sufficient to consider the phase shift after the first pericenter passage in order to determine the boundary for diffusion to happen.

\footnotetext{
${ }^{16}$ We estimate that one would need $\Gamma / \omega_{a} \gtrsim 10^{-6}$ to prevent the parent mode from evolving into the wave-breaking regime $\tilde{E}_{k} \gtrsim 0.1$ by the weakly nonlinear damping as we consider here. While this is much greater than the nonlinear damping rate we find for the $R=1.1 R_{\mathrm{J}}$ model, it can nonetheless be achieved if the Jupiter has a greater radius. For example, we find that a Jupiter model $R=2.0 R_{\mathrm{J}}$ can have $\Gamma / \omega_{a} \simeq 5 \times 10^{-4}$.

17 By comparison, the fractional change in $\Omega_{\text {peri }}$ is only $\mathcal{O}\left(10^{-5}\right)$ as the mode energy grows from 0 to $\tilde{E}_{k} \lesssim 10^{-3}$; see Section 2.2.
}

Specifically, let $\Delta \tilde{E}_{1} \equiv\left|\Delta q_{1}\right|^{2}$ be the energy gained by the mode after the first pericenter passage (suppose it starts with an amplitude $\left.\left|q_{0}\right| \ll\left|\Delta q_{1}\right|\right)$. The phase shift caused by the tidal back-reaction after the first pericenter passage is thus ${ }^{18}$

$$
\Delta \phi_{\mathrm{br}, 1}=-\omega_{a}^{\prime} \Delta P_{\mathrm{orb}, 1}=\frac{3}{2} \omega_{a}^{\prime} P_{\mathrm{orb}, 0} \frac{\Delta \tilde{E}_{1}}{\left|\tilde{E}_{\mathrm{orb}, 0}\right|},
$$

where in the second equality we have used the fact that $\tilde{E}_{\text {orb }, 0}=E_{\text {orb }, 0} / E_{0}<0$. The threshold for growth is approximately $\left|\Delta \phi_{\text {br, } 1}\right| \simeq 1$ rad (Vick \& Lai 2018), which corresponds to a threshold one-kick energy $\Delta \tilde{E}_{1}$ of

$$
\begin{aligned}
\Delta \tilde{E}_{\mathrm{br}, 1} \simeq & 1.0 \times 10^{-5}\left(\frac{\omega_{a}^{\prime}}{\omega_{a}}\right)^{-1}\left(\frac{\omega_{a}}{1.1 \omega_{0}}\right)^{-1}\left(\frac{R}{1.1 R_{\mathrm{J}}}\right)^{5 / 2} \\
& \times\left(\frac{M}{M_{\mathrm{J}}}\right)^{-3 / 2}\left(\frac{M_{*}}{M_{\odot}}\right)^{3 / 2}\left(\frac{a_{\mathrm{orb}, 0}}{\mathrm{au}}\right)^{-5 / 2} \cdot
\end{aligned}
$$

The nonlinear frequency shift also leads to an excess phase of

$$
\Delta \phi_{\mathrm{nl}, 1} \simeq-\left(\Omega \Delta \tilde{E}_{1}\right) P_{\mathrm{orb}, 0}
$$

where the subscript "nl" stands for "nonlinear" effects, and we have used Equation (34) for the nonlinear frequency shift. By setting $\left|\Delta \phi_{\mathrm{nl}, 1}\right|=1 \mathrm{rad}$, we similarly obtain the one-kick energy threshold to trigger diffusive growth solely from nonlinear effects,

$$
\begin{aligned}
\Delta \tilde{E}_{\mathrm{nl}, 1} \simeq & 1.8 \times 10^{-6}\left(\frac{|\Omega|}{30 \omega_{a}}\right)^{-1}\left(\frac{\omega_{a}}{1.1 \omega_{0}}\right)^{-1}\left(\frac{R}{1.1 R_{\mathrm{J}}}\right)^{3 / 2} \\
& \times\left(\frac{M}{M_{\mathrm{J}}}\right)^{-1 / 2}\left(\frac{M_{*}}{M_{\odot}}\right)^{1 / 2}\left(\frac{a_{\mathrm{orb}, 0}}{\mathrm{au}}\right)^{-3 / 2}
\end{aligned}
$$

where we plugged in values representative of the hot Jupiter model described in Section 3.3. Comparing Equations (47) and (49), we see that the nonlinear frequency shift can have a significantly lower one-kick energy threshold than that of tidal back-reaction. It can therefore play a critical role in triggering the diffusive growth of a mode. Furthermore, as we show in Section 3.3, for realistic Jupiter models $\Omega<0$ typically and thus $\Delta \phi_{\mathrm{br}, 1} \Delta \phi_{\mathrm{nl}, 1}>0$. Intuitively, this can be understood as follows. Suppose a positive amount of energy is transferred from the orbit to the mode, and as a result the orbital period decreases. Meanwhile, this energy lowers the frequency at which the mode oscillates as $\Omega<0$ for typical Jupiter models (Table 1). Consequently, both effects make $\omega_{a} P_{\text {orb }}$ decrease. We thus see the two effects add together to further lower the threshold.

\footnotetext{
18 To be consistent with the indexing convention used in Equation (19), we should use $P_{\text {orb }, 1}$ and $\tilde{E}_{\text {orb, } 1}$ in Equations (46) and (48). Nonetheless, using the quantities evaluated at cycle " 0 " will only cause a difference of $\mathcal{O}\left(\Delta \tilde{E}_{1}^{2}\right) \ll 1$, which can be safely ignored.
} 
In order to better see how the relative importance of the two effects scales with the various parameters, we take their ratio

$$
\begin{aligned}
\frac{\left|\Delta \phi_{\mathrm{nl}, 1}\right|}{\left|\Delta \phi_{\mathrm{br}, 1}\right|}= & \frac{2}{3} \frac{|\Omega|}{\omega_{a}} \frac{\omega_{a}}{\omega_{a}^{\prime}} \frac{\left|E_{\mathrm{orb}, 0}\right|}{E_{0}}, \\
= & 5.6\left(\frac{|\Omega|}{30 \omega_{a}}\right)\left(\frac{\omega_{a}^{\prime}}{\omega_{a}}\right)^{-1}\left(\frac{R}{1.1 R_{\mathrm{J}}}\right) \\
& \times\left(\frac{M}{M_{\mathrm{J}}}\right)^{-1}\left(\frac{M_{*}}{M_{\odot}}\right)\left(\frac{a_{\mathrm{orb}, 0}}{\mathrm{au}}\right)^{-1} .
\end{aligned}
$$

Note that the ratio is independent of $\Delta \tilde{E}_{1}$ and $P_{\text {orb,o }}$. Instead, it mainly depends on the ratio of orbital energy to binding energy of the planet, $\sim\left(M_{*} / M\right)\left(R / a_{\text {orb }}\right)$. Consequently, as $a_{\text {orb }}$ decreases during the orbital circularization process, the nonlinear phase shift becomes increasingly dominant over the back-reaction shift. This suggests that the nonlinear frequency shift will play a crucial role in maintaining the diffusive energy transfer from the orbit to the planetary mode in the circularization process.

\subsubsection{Significance of Nonlinear Effects in Other Types of Eccentric Binaries}

We can use Equation (50) to also estimate the significance of the nonlinear effects in other binary systems with highly eccentric orbits. For a binary of solar-type stars in a highly eccentric orbit with $a_{\text {orb }} \sim 1$ au, the nonlinear phase shift is only $\sim 1 \%$ of that due to tidal back-reaction. ${ }^{19} \mathrm{~A}$ similar ratio of a few percent is also found for a neutron star binary with $a_{\text {orb }} \simeq 1000 \mathrm{~km}$ (Vick \& Lai 2019). Indeed, both solar-type stars and neutron stars are more compact (i.e., with smaller $M / R)$ than a typical Jovian planet. Therefore, an eccentric hot Jupiter offers an especially interesting system for studying the impact of nonlinear effects on diffusive growth.

\subsection{Early Orbital Evolution Following the Onset of Diffusive Growth}

In Figure 3 we show a representative example of the first 300 orbits of a Jovian planet orbiting a solar-type star at a semimajor axis $a_{\text {orb }}=1$ au using the iterative map described in Section 4 . The planet's parameters are given in Table 1, and we assume here that the planet is not rotating. In the figure, the pericenter distance is set to $D_{\text {peri }}=3.95 D_{\mathrm{t}}$, where $D_{\mathrm{t}} \equiv R\left(M_{*} / M\right)^{1 / 3} \simeq 11 R_{\mathrm{J}} \simeq$ $5.3 \times 10^{-3}$ au is the tidal radius of the planet. The corresponding eccentricity is thus $e_{\text {orb }}=1-\mathrm{D}_{\text {peri }} / a_{\text {orb }}=0.979$. For these parameters, the one-kick energy is $\Delta \tilde{E}_{1}=\left|\Delta q_{1}\right|^{2}=4.5 \times 10^{-6}$ (Equation (16)). The top panel shows the energy of the parent mode (with $l_{a}=m_{a}=2$ ), and the bottom panel shows the difference of the evolution phase between two adjacent cycles (Equation (45)). We see that in the linear case (gray trace) the difference in the mode's propagation phase between adjacent cycles, $\left|\Delta \phi_{\mathrm{k}}\right|$, is small $(\sim 0.1 \mathrm{rad})$, and the mode energy just undergoes periodic oscillations (see also the discussions in Vick \& Lai 2018). By contrast, when we include nonlinear mode interactions, there is an additional contribution to the random phase due to the nonlinear frequency shift (Equation (48)). As a

\footnotetext{
19 This is specifically for the nonresonant nonlinear effect of the $l_{a}=2 f$-mode. A solar-type star also has low-frequency $g$-modes that have very different values of $\Omega$ from that of the $f$-mode. Those $g$-modes may allow for a richer family of nonlinear effects, such as the parametric instability.
}

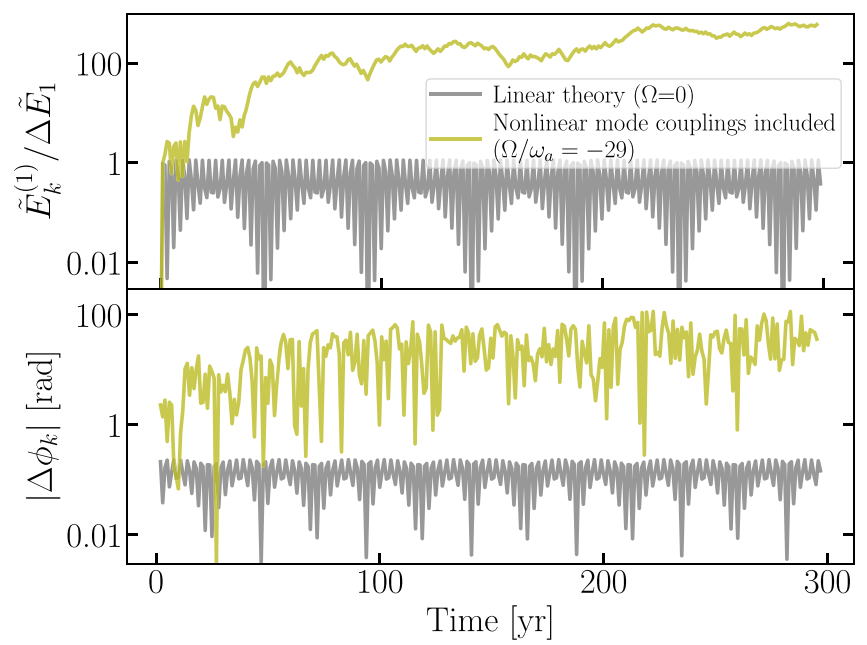

Figure 3. Evolution trajectories during the first 300 orbits. The top panel shows the mode energy relative to the one-kick energy, and the bottom panel shows the difference in the mode's excess phase between the $k$ th and $(k-1)$ th orbit. We fix the pericenter distance at $D_{\text {peri }}=3.95 D_{\mathrm{t}}$ and assume that the planet is nonrotating, resulting in a one-kick energy $\Delta \tilde{E}_{1}=4.5 \times 10^{-6}$. In the linear case $(\Omega=0$; gray lines) the mode energy and excess phase oscillate periodically and there is no diffusive growth. However, when nonlinear mode interactions are included $\left(\Omega / \omega_{a}=-29\right.$; olive lines), the excess phase is significantly larger and varies randomly, resulting in diffusive growth of the $f$-mode's energy.

result, we see that the $f$-mode's energy grows diffusively, unlike in the linear case. After 300 cycles, the mode energy grows to about $300 \Delta \tilde{E}_{1}$, as one would expect for a diffusive process (i.e., the amplitude grows as the square root of the number of pericenter kicks).

In Figure 4 we systematically explore some of the conditions necessary to trigger diffusive growth. We show the maximum mode energy achieved after 500 orbital cycles (about $500 \mathrm{yr}$ ) as a function of the pericenter distance (or, equivalently, the orbital eccentricity since $D_{\text {peri }}=\mathrm{a}_{\text {orb }}\left(1-e_{\text {orb }}\right)$, and we set the initial semimajor axis at $a_{\mathrm{orb}, 0}=1 \mathrm{au}$ ). For the given planetary model (Table 1), the one-kick energy $\Delta \tilde{E}_{1}$ is shown in the top $x$-axis for each choice of $D_{\text {peri. }}$. In the left panel, we assume that the planet is nonrotating, while in the right panel we assume that it is pseudosynchronized with the orbit with $\Omega_{\mathrm{s}} / \omega_{a}=0.19\left(D_{\text {peri }} / 0.02 \text { au }\right)^{-3 / 2}$ and $\omega_{a}^{\prime}=\omega_{a}+2 \Omega_{\mathrm{s}}$. If a mode experiences diffusive growth, then its energy after $k$ pericenter passages is expected to be $\tilde{E}_{\mathrm{k}} \sim k \Delta \tilde{E}_{1}$ on average. Since we set $k=500$, we expect $\max \left[\tilde{E}_{k}\right] \simeq$ $500 \Delta \tilde{E}_{1}$ for a mode that grows diffusively (indicated by the black lines), while $\max \left[\tilde{E}_{k}\right] \sim \Delta \tilde{E}_{1}$ for a mode that does not grow (assuming that the mode is off resonance with the orbit; in the right panel $\omega_{a}^{\prime}$ changes as we vary $D_{\text {peri }}$, allowing it to scan through a series of resonances with different orbital harmonics, thereby causing the excess features to the right of the vertical lines, which we will discuss in Section 5.3).

We see that each panel in Figure 4 can be divided up into two regions according to the maximum mode energy achieved. Let us first focus on the left panel (a nonrotating planet with $\omega_{a}$ $P_{\text {orb, } 0} / 2 \pi=2818.73$ being a noninteger). In the linear case $(\Omega=0$ ), we find numerically that the boundary where diffusive growth is first triggered is at $D_{\text {peri }} \simeq 3.75 D_{\mathrm{t}}$, corresponding to a one-kick energy of $\Delta \tilde{E}_{1} \simeq 1.4 \times 10^{-5}$. The analytical estimate (Equation (47); see vertical gray line) agrees well with the numerical results but slightly overestimates the threshold value of $D_{\text {peri }}$ (and underestimates $\Delta \tilde{E}_{1}$ ) because there we simply 


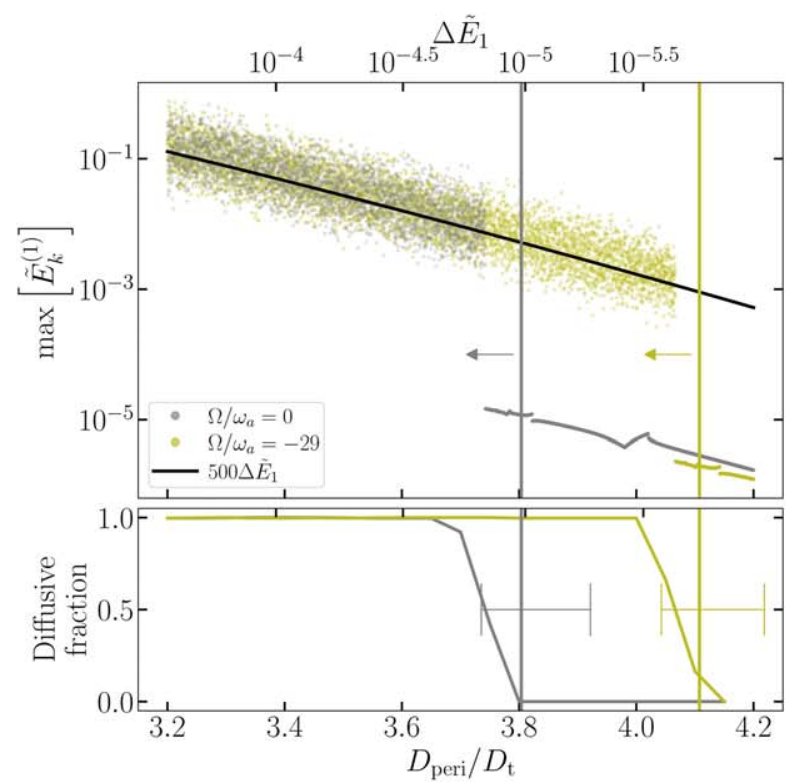

(a) Non-spinning.

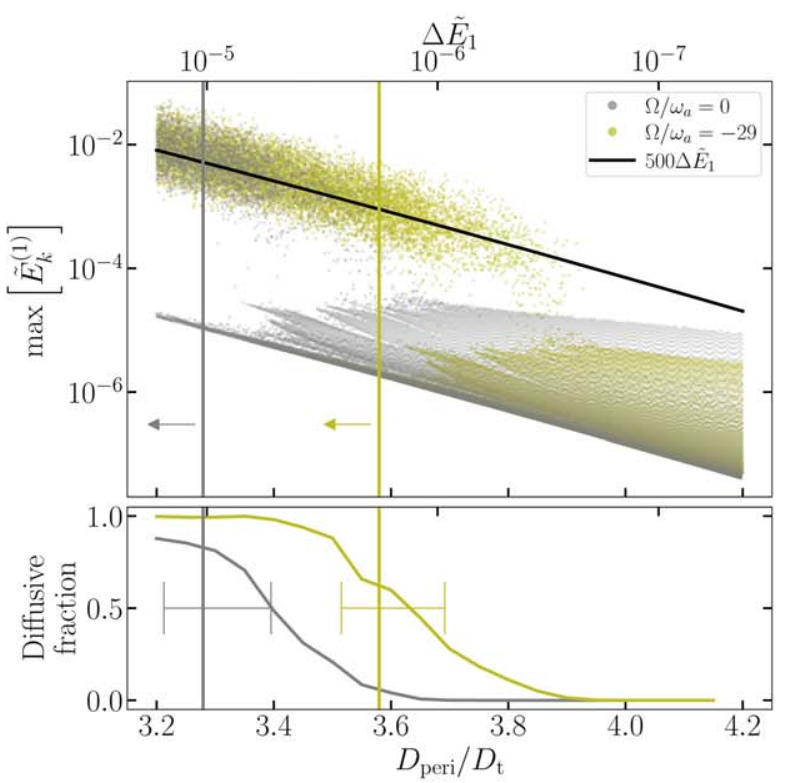

(b) Pseudo-synchronized.

Figure 4. Top panels: maximum $f$-mode energy achieved after 500 pericenter passages. The gray circles are calculated assuming linear theory, while the olive circles also include nonlinear mode interactions (with $\Omega / \omega_{a}=-29$ ). In both plots we set $a_{\text {orb }, 0}=1$ au. In the left panel we assume that the planet is not spinning, while in the right panel we assume that it spins at a constant rate given by the pseudo-synchronization condition (Equation (23)). Bottom panels: the fraction of systems that undergoes the diffusive growth (i.e., points around the black lines) as a function of $D_{\text {peri, }}$, estimated over a full bin width of $0.1 D_{\mathrm{t}}$. The vertical lines are the analytic estimates for the diffusive growth threshold based on Equations (47) and (49) by setting $\Delta \phi_{1}=1 \mathrm{rad}$. The error bars are obtained if we instead use $\Delta \phi_{1}=0.5$ or $1.5 \mathrm{rad}$. The regions where we expect diffusive growth to occur are also indicated by arrows in the top panel. In the right panel, a mode to the right of the vertical lines can occasionally grow to an intermediate amplitude of $\tilde{E}_{k}^{(1)} \simeq$ a few $\times 10^{-5}$ if its frequency $\omega_{a}^{\prime}$ comes close to resonance with one of the orbital harmonics.

assumed the threshold phase shift to be $1 \mathrm{rad}$; in reality a slightly greater phase shift is required. This can also be seen from the bottom panel, where we show the fraction of systems undergoing diffusive growth (i.e., the fraction of points around the black lines; the estimate is performed over a full bin width of $0.1 D_{\mathrm{t}}$ ). The error bars around the vertical lines are obtained by setting $\Delta \phi_{1}=0.5$ and $1.5 \mathrm{rad}$ and then reevaluating $\Delta \tilde{E}_{1}$ using Equations (46) and (48).

When we account for the nonlinear frequency shift, we find that the boundary moves to larger $D_{\text {peri }}$ (smaller one-kick energies). For the representative value of $\Omega / \omega_{a} \simeq-29$ (see Table 1), we find that the threshold one-kick energy is lowered to $\Delta \tilde{E}_{1} \simeq 2.4 \times 10^{-6}$, which is a factor of about 6 smaller than the linear case (see the vertical olive line and Equation (49); note that the threshold is in fact determined by the sum $\Delta \phi_{\mathrm{nl}, 1}+\Delta \phi_{\mathrm{br}, 1}$, with the latter being $\simeq 20 \%$ of the former for the parameters in Figure 4). Because the one-kick energy depends sensitively on the pericenter distance, a factor of six change in $\Delta \tilde{E}_{1}$ corresponds to a $\simeq 10 \%$ increase in $D_{\text {peri }}$.

\subsection{Including Spin Effects}

We consider the effects of planet spin in the right panel of Figure 4 . We assume that the planet is rotating at a rate determined by the pseudo-synchronization condition (Equation (23)). ${ }^{20}$ As we show in Section 2.1, the mapping equations including spin are formally the same as the nonspinning equations except that the mode frequency is replaced by the inertial-frame value $\omega_{a}^{\prime}=\omega_{a}+m_{a} \Omega_{\mathrm{s}}$ (this frequency then enters the calculations of the one-kick amplitude and the linear propagation phase).

\footnotetext{
${ }^{20}$ We assume pseudo-synchronization here as a plausible scenario. Whether it can be achieved through, e.g., a Lidov-Kozai evolution involving a tertiary mass remains to be answered by future studies.
}

Since we focus on a prograde mode with $m_{a}=2,\left|\Delta q_{1}\right| \propto K_{22}$ decreases sharply as $\omega_{a}^{\prime}$ increases (Equation (15)). As a result, the $D_{\text {peri }}$ boundary where diffusive growth is first triggered is shifted to smaller values.

At the same time, in the right panel the mode can occasionally become resonant with the orbit when $\omega_{a}^{\prime} P_{\text {orb }} / 2 \pi=$ integer, as $\omega_{a}^{\prime}$ now varies with $D_{\text {peri }}$ owing to the pseudo-synchronization condition (this is in contrast to the left panel, where $\omega_{a} P_{\text {orb }} / 2 \pi$ is fixed at a noninteger value when we vary $\left.D_{\text {peri }}\right)$. For $D_{\text {peri }} / \mathrm{D}_{\mathrm{t}} \gtrsim 3.8$, such resonances can bring the mode energy up to $\tilde{E}_{k}^{(1)} \simeq$ a few $\times 10^{-5} \gg \Delta \tilde{E}_{1}$. However, as the mode acquires energy from the orbit, the orbital period starts to change (though not by a significant enough amount to trigger diffusion). It thus destroys the resonance and prevents the mode energy from increasing further (see also Vick \& Lai 2018). Similarly, the nonlinear frequency shift also destroys the resonance between $\omega_{a}^{\prime}$ and $P_{\mathrm{orb}}$, and this is why the upper envelope of the olive circles is at a lower value than that of the gray ones.

At $3.2 \lesssim D_{\text {peri }} / D_{\mathrm{t}} \lesssim 3.8$, we see that the chance resonance with the orbit may occasionally help a slightly subthreshold mode to also evolve into the diffusive regime. Suppose that the chance resonance helps the mode to initially build up an energy $\tilde{E}_{k, 0} \simeq 10^{-5}\left(10^{-4}\right)$ with (without) the nonlinear effect (corresponding approximately to the upper envelopes shown in the right panel). The typical energy exchange between a mode and the orbit is then given by $\sqrt{\tilde{E}_{k, 0} \Delta \tilde{E}_{1}} \gg \Delta \tilde{E}_{1}$ (Wu 2018). If we replace $\Delta \tilde{E}_{1}$ by $\sqrt{\tilde{E}_{k, 0} \Delta \tilde{E}_{1}}$ in Equations (46) and (48), we see that the new threshold one-kick energy becomes $\Delta \tilde{E}_{b r, 1} \simeq 10^{-6}$ and $\Delta \tilde{E}_{n l, 1} \simeq 3 \times 10^{-7}$ for modes that are initially in close resonance with the orbit. As $D_{\text {peri }}$ decreases and $\Delta \tilde{E}_{1}$ increases, even systems that are not at the upper envelope may enter the chaotic regime, and the fraction of diffusive systems increases 


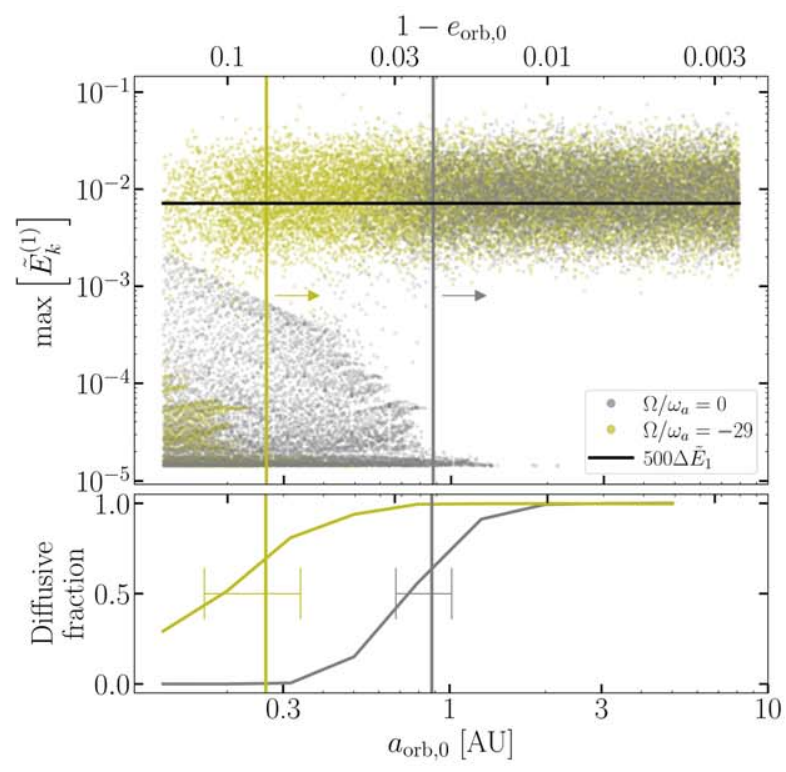

(a) Non-spinning.

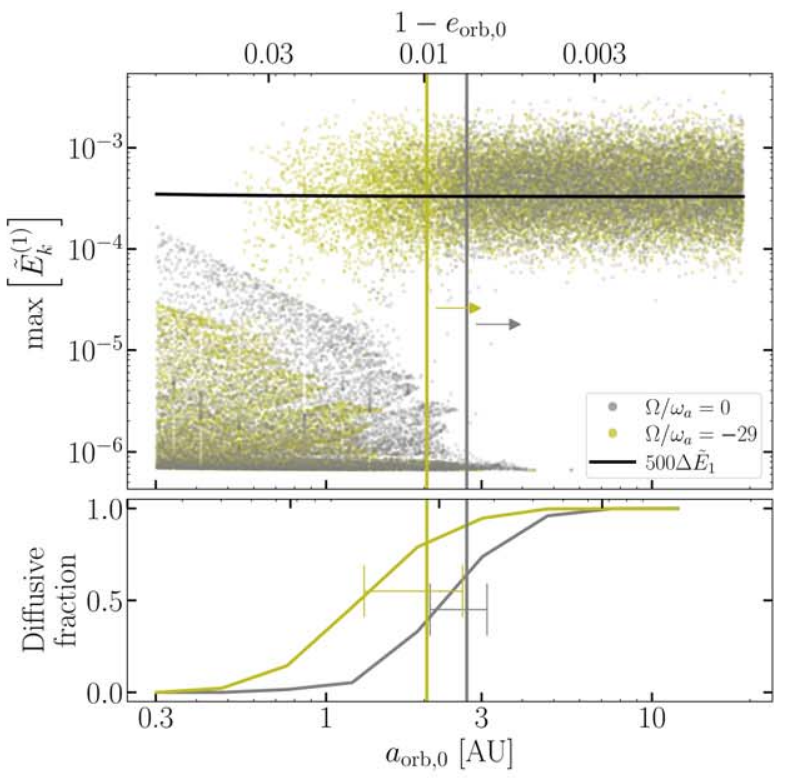

(b) Pseudo-synchronized.

Figure 5. Similar to Figure 4, but this time we fix the pericenter distance at $D_{\text {peri }}=0.02$ au and let the initial semimajor axis $a_{\text {orb,o }}$ vary (the top axis of each panel shows the corresponding $\left.1-e_{\mathrm{orb}, 0}\right)$. The gray and olive vertical lines are calculated using Equations (51) and (52), respectively, assuming that $\Delta \phi_{1}=1$ rad is needed to trigger diffusive growth; the error bars show the threshold if instead $\Delta \phi_{1}=0.5$ or 1.5 rad is needed. The fraction of systems undergoing diffusive growth shown in the bottom panel is estimated over logarithmic bins with full width of $\log _{10}\left(a_{\mathrm{orb}, 0} / \mathrm{au}\right)=0.4$. A system with an $a_{\mathrm{orb}, 0}$ that is slightly below the threshold may still trigger diffusive growth if the mode is close to resonance with the orbit.

as indicated by the bottom panel. Eventually, when $\Delta \tilde{E}_{1}$ reaches the value derived in Equations (47) and (49), almost all of the systems will grow diffusively.

\subsection{Threshold Expressed in Terms of Semimajor Axis Rather Than One-kick Energy}

An alternative way to consider the problem is to hold $D_{\text {peri }}$ and thus $\Delta \tilde{E}_{1}$ fixed and instead vary the initial semimajor axis $a_{\mathrm{orb}, 0}=\mathrm{D}_{\text {peri }} /\left(1-e_{\mathrm{orb}, 0}\right)$. By setting $\left|\Delta \phi_{\mathrm{br}, 1}\right|=1 \mathrm{rad}$ in Equation (46) as before (Section 5.1) but now solving for $a_{\mathrm{orb}, 0}^{(\mathrm{br})}$, we find that the threshold to trigger diffusive growth due to only tidal back-reaction is

$$
\begin{aligned}
a_{\mathrm{orb}, 0}^{(\mathrm{br})} \simeq & 2.5 \mathrm{au}\left(\frac{\Delta \tilde{E}_{1}}{10^{-6}}\right)^{-2 / 5}\left(\frac{\omega_{a}^{\prime}}{\omega_{a}}\right)^{-2 / 5}\left(\frac{\omega_{a}}{1.1 \omega_{0}}\right)^{-2 / 5} \\
& \times\left(\frac{R}{1.1 R_{\mathrm{J}}}\right)\left(\frac{M}{M_{\mathrm{J}}}\right)^{-3 / 5}\left(\frac{M_{*}}{M_{\odot}}\right)^{3 / 5} .
\end{aligned}
$$

Similarly, we can use Equation (48) to find the threshold due to nonlinear mode interactions

$$
\begin{aligned}
a_{\mathrm{orb}, 0}^{(\mathrm{nl})} \simeq & 1.5 \mathrm{au}\left(\frac{\Delta \tilde{E}_{1}}{10^{-6}}\right)^{-2 / 3}\left(\frac{|\Omega|}{30 \omega_{a}}\right)^{-2 / 3}\left(\frac{\omega_{a}}{1.1 \omega_{0}}\right)^{-2 / 3} \\
& \times\left(\frac{R}{1.1 R_{\mathrm{J}}}\right)\left(\frac{M}{M_{\mathrm{J}}}\right)^{-1 / 3}\left(\frac{M_{*}}{M_{\odot}}\right)^{1 / 3} .
\end{aligned}
$$

In both cases, the threshold $a_{\text {orb, } 0}$ increases with decreasing one-kick energy $\Delta \tilde{E}_{1}$ (i.e., increasing $D_{\text {peri }}$ ). This is because both $\Delta \phi_{\mathrm{br}}$ and $\Delta \phi_{\mathrm{nl}} \propto P_{\text {orb }} \Delta \tilde{E}_{1}$ (Equations (46) and (48)), and a longer $P_{\mathrm{orb}} \propto a_{\mathrm{orb}}^{3 / 2}$ is thus required in order for the $f$-mode to accumulate an excess phase $\left|\Delta \phi_{k}\right|$ to $\mathcal{O}(1) \mathrm{rad}$. Additionally, Equations (51) and (52) scale differently with $\Delta \tilde{E}_{1}$ because
$\Delta \phi_{\mathrm{br}} \propto\left|E_{\mathrm{orb}}\right|^{-1}$, which reflects the fact that an orbit with greater $a_{\text {orb }}$ is less bound and thus sees a greater change in the fractional orbital period. Also note that Equation (52) overestimates the minimum $a_{\mathrm{orb}, 0}$ required to trigger the diffusion because it assumes only the nonlinear contribution to $\Delta \phi_{\mathrm{k}}$. In reality, the nonlinear frequency shift and the tidal back-reaction both contribute to $\Delta \phi_{\mathrm{k}}$, and they have the same sign (Section 5.1). Similar to the case shown in the right panel of Figure 4, Equations (51) and(52) should be treated as the upper end of the thresholds; almost all of the systems with $a_{\text {orb }, 0}$ greater than the values estimated in Equations (51) and (52) will trigger the diffusive evolution. On the other hand, if a system is initially close to being resonant with the orbit, then at smaller $a_{\text {orb, } 0}$ it may still enter the diffusive regime.

We evaluate the boundary in $a_{\mathrm{orb}, 0}$ numerically in Figure 5 . Here we fix the pericenter distance to be $D_{\text {peri }}=0.02$ au, corresponding to a one-kick energy of $\Delta \tilde{E}_{1} \simeq 1.4 \times 10^{-5}$ $\left(\Delta \tilde{E}_{1} \simeq 6.7 \times 10^{-7}\right)$ for a nonspinning (pseudo-synchronized) planet. The situation is particularly interesting astrophysically in the case where the planet's spin is pseudo-synchronized. For a relatively weak one-kick energy of $\Delta \tilde{E}_{1} \lesssim 10^{-6}$, only planets born $\gtrsim 2$ au away from the host star can trigger diffusive tidal evolution and form hot Jupiters if only the linear theory is used. On the other hand, when we include nonlinear mode interactions, it can be triggered for planets born with $a_{\mathrm{orb}, 0} \simeq 0.7-2$ au. Thus, nonlinear mode interactions significantly expand the parameter space allowed for diffusive growth to happen, which not only allows more potential progenitors to form hot Jupiters within the age of the universe but also saves more planets from disruption by the host star during the Kozai cycles (see, e.g., Vick et al. 2019). It could thus help alleviate the discrepancy between the predicted and observed hot Jupiter/regular Jupiter occurrence rate (see Dawson \& Johnson 2018). 


\section{Conclusion and Discussion}

We studied the nonlinear interaction between a self-coupled parent $f$-mode and daughter $f$ - and $p$-modes in a Jovian planet. For a parent mode with azimuthal quantum number $m_{a}=2$ and frequency $\omega_{a}$, it drives both $m_{b}=-4$ daughters that correspond to waves oscillating at $2 \omega_{a}$ and nonoscillatory $m_{c}=0$ daughters that correspond to a modification of the planet's structure (Section 3). We found that at leading order the interaction leads to a nonlinear shift in the parent mode's eigenfrequency, $\delta \omega_{a}$, as well as a nonlinear increase in the parent mode's damping rate (imaginary part of the frequency), $\delta \gamma_{a}$. Both the nonlinear frequency shift and damping rate follow the scaling $\delta \omega_{a}$, $\delta \gamma_{a} \propto \omega_{a} E_{a}$ at leading order (Equations (34) and (35); see also Kumar et al. 1994; Kumar \& Goodman 1996). The modifications are time dependent because we consider planets on highly eccentric orbits with parent mode energies $E_{a}$ that vary at each pericenter passage. Furthermore, we showed that although the frequency shift can, in principle, be either positive or negative, for typical Jupiter models a negative shift is more likely (that is, the parent mode's eigenfrequency decreases with increasing mode energy). The nonlinear damping, on the other hand, strictly increases as the mode energy increases (see Table 1 and Figure 2).

We then developed the formalism to construct iterative maps including nonlinear effects and applied them to study how nonlinear interactions affect the high-eccentricity migration of proto-hot Jupiters. We found that the energy-dependent nonlinear frequency shift leads to an excess phase of the parent mode (Equation (48)), which is stochastic from orbit to orbit. It thus provides another channel for triggering the diffusive growth of the parent mode in addition to the tidal back-reaction considered in previous studies.

In fact, we found that for typical Jupiter models the nonlinear phase shift is $\approx 5$ times larger than the phase shift due to backreaction (Equation (50)). The two effects add together and lower the threshold one-kick energy required in order to trigger the growth by about a factor of $\approx 6$ compared to the case without nonlinear interactions (Figure 4). Alternatively, if one fixes the one-kick energy, the threshold on the minimum initial orbital semimajor axis can be lowered by a factor of $\approx 2$ (Figure 5). If the one-kick energy is small (due to either a small eccentricity and hence large pericenter distance or a high spin rate of the planet), then in the linear case only planets born at $a_{\mathrm{orb}, 0} \gtrsim 2$ au can undergo diffusive tidal evolution and form hot Jupiters; however, when nonlinear interactions are accounted for, it is lowered to the interesting range of $a_{\mathrm{orb}, 0}=0.7-2 \mathrm{au}$.

In this paper, we focused on developing the theoretical framework and considered only the evolution over the first $\mathcal{O}(100) \mathrm{yr}$. There are several aspects of the problem we think would be interesting to address in future studies.

First, what is the long-term evolution of the system over $\sim 10$ kyr? For the Jupiter model we considered in this work (Table 1), the weakly nonlinear damping $\propto \Gamma$ is weak, and thus the parent mode energy will grow so large that it likely becomes strongly nonlinear, as assumed by $\mathrm{Wu}$ (but see discussion below). Wu found that the diffusive process, and hence the orbital evolution, typically stalls when the semimajor axis decays to $a_{\text {orb }} \simeq 0.2$ au while the eccentricity is still high $\left(e_{\text {orb }} \simeq 0.9-0.95\right)$, and it was unclear what drives the subsequent orbital circularization.
On the other hand, we estimate that the enhancements due to nonlinear mode interactions may help drive the circularization down to $e_{\text {orb }} \simeq 0.8-0.85$ (we plan to study this more fully in future work). Note that one can still estimate the termination point by setting Equations (46) and (48) to $\simeq 1 \mathrm{rad,} \mathrm{with} \Delta \tilde{E}_{1}$ replaced by $\sqrt{\tilde{E}_{k} \Delta \tilde{E}_{1}}$ and $P_{\mathrm{orb}, 0}$ and $\tilde{E}_{\mathrm{orb}, 0}$ replaced by their current values $P_{\text {orb }, k}$ and $\tilde{E}_{\text {orb }, k}$. It is easy to show that the magnitude of the random phase induced by nonlinear interaction decreases slower than that due to the tidal backreaction as the orbit decays; see Equation (50). Physically, this is because the orbit "hardens" ( $\left|E_{\text {orb }}\right|$ increases $)$ as $a_{\text {orb }}$ shrinks, which makes it increasingly hard to be perturbed by the tidal back-reaction (Equations (19) and (46)). By contrast, the natural energy scale that enters the nonlinear phase shift is the

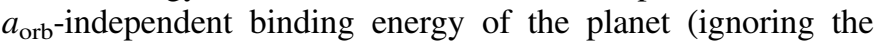
evolution of the planet). An efficient circularization could help explain both the paucity of supereccentric Jupiters (Socrates et al. 2012) and the relatively young age of hot Jupiter host stars (Hamer \& Schlaufman 2019).

It would also be interesting to investigate how the values of $(\Omega, \Gamma)$ vary for different Jupiter models and how the tidal evolution trajectories depend on $(\Omega, \Gamma)$. We estimate that if $\Gamma \gtrsim 10^{-6} \omega_{a}$, weakly nonlinear damping could be sufficient to prevent the parent mode from evolving into the strongly nonlinear regime. This would lead to another qualitative difference from the trajectories found in $\mathrm{Wu}$ (2018), in addition to the excess nonlinear phase shift discussed above. Such large dissipation rates can be achieved by Jupiter models with greater radii, and this could have potentially important observational consequences.

A calculation that combines diffusive tidal evolution with the mechanism that drives the eccentricity to large values in the first place (e.g., Lidov-Kozai cycles) would be valuable and help test these ideas further. By including nonlinear effects, it would extend the work of Vick et al. (2019) and thereby provide a more robust estimate of the formation rate of hot Jupiters due to diffusive tidal evolution. Current theories produce too few hot Jupiters relative to regular Jupiters, and it would be interesting to know whether nonlinear effects could help mitigate the tension.

To carry out the studies described above, a few modifications to the current framework would be needed. For instance, as the parent mode's energy builds up and its eigenfrequency decreases, the orbital integral $K_{l m}$ should be modified accordingly. Since the parent's frequency is typically shifted to a lower value $(\Omega<0)$, we would expect the one-kick amplitude $\Delta q_{1} \propto K_{l m}$ to increase in magnitude as the parent's energy increases (Equation (15)). This would further enhance the significance of the nonlinear effects. On the other hand, we do not expect a linear-in-energy frequency shift (Equation (34)) to be accurate when $|\Omega| \tilde{E}_{a} \simeq \omega_{a}$. Note that this condition can happen at a smaller energy than the wave-breaking energy, and therefore further corrections would be needed.

We thank Dong Lai, Jim Fuller, Yanqin Wu, Fei Dai, Heather Knutson, and the anonymous referee for helpful discussions and comments during the conception and the development of this study. This work was supported by NSF AST-2054353. H.Y. acknowledges the support of the Sherman Fairchild Foundation. 


\section{Appendix \\ Amplitude of $\boldsymbol{m} \neq \mathbf{0}$ Daughters}

In Equations (A4) and (30) we assumed that the daughter modes' amplitudes are given by their steady-state values. While this is a good approximation for "mode $c$ " (daughters with $m_{c}=0$ ), as we explain here the problem may be more involved for "mode $b$ " (daughters with $l_{b}=-m_{b}=4$ ).

The equation governing such a mode $b$ is given by (see Equation (25))

$$
\dot{q}_{b}+\left(i \omega_{b}+\gamma_{b}\right) q_{b}=i \omega_{b} \kappa_{b}\left(q_{a}^{*} q_{a}^{*}-2 q_{a}^{*} U_{a}^{*}\right),
$$

where, as explained in Section 3.1 (also see discussion below), we can ignore the linear tidal forcing on mode $b$, i.e., the $U_{b}$ term. We can decompose the parent mode (mode $a$ ) as

$$
q_{a}=q_{a, \mathrm{dyn}}+q_{a, \mathrm{eq}}=q_{a, \mathrm{dyn}}+U_{a},
$$

where $q_{a \text {,eq }} \equiv U_{a}$ is the equilibrium tide solution of mode $a$ (which can be obtained from Equation (24) when we ignore the nonlinear couplings and treat $\left.\left|\dot{q}_{a}\right|,\left|\gamma_{a} q_{a}\right| \ll\left|\omega_{a} q_{a}\right|\right)$. We thus have

$$
\dot{q}_{b}+\left(i \omega_{b}+\gamma_{b}\right) q_{b}=i \omega_{b} \kappa_{b}\left(q_{a, \mathrm{dyn}}^{*} q_{a, \mathrm{dyn}}^{*}+U_{a}^{*} U_{a}^{*}\right) .
$$

In the main text, we focused on the steady-state solution of $q_{b}$ driven by a free-oscillating parent. That is, we assumed that $q_{a}$ contains only the dynamical component $q_{a, \mathrm{dyn}}$, which oscillates at a single frequency $\omega_{a}$, and found (see Equation (29))

$$
q_{b, \mathrm{ss}}=\frac{\omega_{b} \kappa_{b}}{\Delta_{b}-i \gamma_{b}}\left(q_{a, \mathrm{dyn}}^{*}\right)^{2},
$$

where $\Delta_{b}=\left(\omega_{b}+2 \omega_{a}\right)$. Note, however, that the steady-state solution $q_{b, \mathrm{ss}}$ neglects the $U_{a}^{*} U_{a}^{*}$ term in Equation (A3) and neglects the "transient" part of the solution for $q_{b}$. We will refer to the latter as the history term since it depends on the past history of $q_{b}(t)$ from previous pericenter passages. Here we will show that the $U_{a}^{*} U_{a}^{*}$ term should always be insignificant, but not necessarily the history term.

We will make two simplifications in our analysis. First, we do not explicitly solve for the instantaneous value of $q_{b}$ in the vicinity of a pericenter passage for simplicity. As we will see, this does not preclude us from obtaining a qualitative estimate of the history term due to previous pericenter passages. Second, we treat the parent mode as if it is unperturbed by nonlinear interactions. As a result, we assume that the dynamical component of the parent mode, $q_{a, \mathrm{dyn}}$, when far away from the pericenter, oscillates at $\omega_{a}$ and not at the nonlinearly shifted frequency (see Section 3.1).

\section{A.1. The $U_{a}^{*} U_{a}^{*}$ Term}

First, we consider the drive due to the $U_{a}^{*} U_{a}^{*}$ term. Similar to the one-kick amplitude of the parent $\Delta q_{a, 1}$, we can define a one-kick amplitude of mode $b$ at each pericenter passage due to the $U_{a}^{*} U_{a}^{*}$ term as

$$
\begin{aligned}
\left(\Delta q_{b, 1}\right)_{U_{a}^{2}}= & \int i \omega_{b} \kappa_{b} W_{l_{a} m_{a}}^{2} \\
& \times Q_{a}^{2}\left(\frac{M_{*}}{M}\right)^{2}\left(\frac{R}{D}\right)^{l_{b}+2} e^{i\left[\left(\omega_{b}+m_{b} \Omega_{\mathrm{s}}\right) \tau-m_{b} \Phi\right]},
\end{aligned}
$$

where we used the fact that $l_{b}=2 l_{a}$ and $m_{b}=-2 m_{a}$. If we define

$$
K_{l m}^{\prime}(\omega)=\frac{\omega_{0}}{2 \pi} \int\left[\frac{D_{\text {peri }}}{D(\tau)}\right]^{l+1} e^{i[\omega \tau-m \Phi(\tau)]} d \tau,
$$

as a modified temporal overlap, then we can write the one-kick amplitude of the daughter mode as

$$
\begin{aligned}
\left(\Delta q_{b, 1}\right)_{U_{a}^{2}}= & i 2 \pi \kappa_{b} W_{22}^{2} Q_{a}^{2}\left(\frac{\omega_{b}}{\omega_{0}}\right)\left(\frac{M_{*}}{M}\right)^{2} \\
& \times\left(\frac{R}{D_{\text {peri }}}\right)^{6} K_{5,-4}^{\prime}\left(\omega_{b}-4 \Omega_{\mathrm{s}}\right),
\end{aligned}
$$

where we have plugged in $l_{a}=m_{a}=2$ for the parent and $l_{b}=-m_{b}=4$ for the daughter. For typical values $\left(a_{\mathrm{orb}}=1 \mathrm{au}\right.$, $e_{\text {orb }}=0.98, \omega_{a}=5.6 \times 10^{-4} \mathrm{rad} \mathrm{s}^{-1}$, and $\left.\omega_{b}=-1.9 \omega_{a}\right)$, we find $\left|\left(\Delta q_{b}\right)_{U_{a}^{2}}\right| \simeq 0.4\left|\Delta q_{a, 1}^{2}\right|$.

Although initially $\left(\Delta q_{b, 1}\right)_{U_{a}^{2}}$ is comparable to the steady-state solution $q_{b, \mathrm{ss}}$ (Equation (A4)), as the system starts to grow diffusively, its effect will soon become subdominant. This can be seen by noticing that even if $\left(\Delta q_{b, 1}\right)_{U_{a}^{2}}$ can grow diffusively itself (e.g., due to a random $P_{\text {orb }}$ ), after $k$ pericenter passages it only increases the amplitude of $q_{b}$ by $\sqrt{k}\left|\left(\Delta q_{b, 1}\right)_{U_{a}^{2}}\right| \propto k^{1 / 2}$ on average. On the other hand, $q_{b, \mathrm{ss}} \sim k\left|q_{a, \mathrm{dyn}}\right|^{2} \propto k$ because each $\left|q_{a, \text { dyn }}\right|$ grows as $\sqrt{k}$. Consequently, the significance of the $q_{b, \mathrm{ss}}$ term increases as $\sqrt{k}$. In fact, the dominance of $q_{b, \mathrm{ss}}$ is further enhanced by the $\omega_{b} / \Delta_{b}$ factor, especially for the most resonant daughter mode with the smallest $\left|2 \omega_{a}+\omega_{b}\right|$.

We therefore conclude that the modification to $q_{b}$ due to the $\left(U_{a}^{*}\right)^{2}$ term can be ignored. It is also worth noting that the drive from $\left(U_{a}^{*}\right)^{2}$ is stronger than the direct tidal force on mode $b, \quad U_{b} \propto\left(M_{*} / M\right)\left(R / D_{\text {peri }}\right)^{l_{b}+1}$, by a factor of $\left(M_{*} / M\right)\left(R / D_{\text {peri }}\right)\left(\kappa_{b} W_{22}^{2} Q_{a}^{2} / W_{44} Q_{b}\right) \simeq 6\left(\kappa_{b} W_{22}^{2} Q_{a}^{2} / W_{44} Q_{b}\right)$. It thus justifies why we can also ignore the daughter modes' linear coupling to the tide.

$$
\text { A.2. The } q_{a, \mathrm{dyn}}^{*} q_{a, \mathrm{dyn}}^{*} \text { Term }
$$

We now consider the effect of the history term on the daughter.

If we define $c_{b}=q_{b} \exp \left(-2 i \omega_{a} t\right)$, then by Equation (A3)

$$
\dot{c}_{b}+\left(i \Delta_{b}+\gamma_{b}\right) c_{b}=i \omega_{b} V_{b}
$$

where $V_{b} \equiv \kappa_{b} q_{a}^{*} q_{a}^{*} \exp \left(-2 i \omega_{a} t\right)$. Our definition of $V_{b}$ does not include the equilibrium tide contribution $U_{a}^{*} U_{a}^{*}$ and $U_{b}$ since we showed above that they are insignificant. For the same reason, here and below we drop the "dyn" subscript on the parent. Note that if we ignore the parent's nonlinear frequency corrections, then away from pericenter $q_{a} \sim e^{-i \omega_{a} t}$, and thus $V_{b}$ is a constant. Near pericenter, however, $q_{a}$ has an additional time dependence owing to the kick $\Delta q_{a, 1}$ the parent receives over a timescale $1 / \Omega_{\text {peri. }}$. As we will see, it is this effect that constitutes the history term we are interested in. 
The general solution for $c_{b}$ is given by

$$
\begin{aligned}
c_{b}(t)= & e^{-\left(i \Delta_{b}+\gamma_{b}\right) t} \int_{t_{0}}^{t} i \omega_{b} V_{b}(\tau) e^{\left(i \Delta_{b}+\gamma_{b}\right) \tau} d \tau . \\
= & \left.\frac{\omega_{b}}{\Delta_{b}-i \gamma} V_{b}\right|_{t_{0}} ^{t}-e^{-\left(i \Delta_{b}+\gamma_{b}\right) t} \omega_{b} \\
& \times \int_{t_{0}}^{t} \frac{\dot{V}_{b}(\tau)}{\Delta_{b}-i \gamma_{b}} e^{\left(i \Delta_{b}+\gamma_{b}\right) \tau} d \tau,
\end{aligned}
$$

where the initial time is $t_{0}$ and we performed integration by parts to get the second line. For future convenience we set $V_{b}\left(t_{0}\right)=0$ and thus drop the initial condition. Note that the first term in Equation (A9) recovers the steady-state solution, Equation (29), and it depends only on the instantaneous value of $q_{a}$. The second term, on the other hand, captures the past history. For a free oscillator, $q_{a} \sim e^{-i \omega_{a} t}$ and $\dot{V}_{b}=0$, and thus the value of $q_{b}$ is independent of the past history.

When the system is coupled to the tide, however, we have $\dot{V}_{b} \sim \Omega_{\text {peri }} V_{b}$ in the vicinity of the pericenter. First, consider a mode $b$ for which $\left|\Delta_{b}\right| \gg \Omega_{\text {peri }} \gg \gamma_{b}$. These inequalities hold for all the daughters in our mode networks with the exception of the $l_{b}=-m_{b}=4, \omega_{b}<0 f$-mode, which we consider separately below. For large detuning, if we keep performing integration by parts, we get

$$
\begin{aligned}
c_{b}(t) \simeq & \frac{\omega_{b}}{\Delta_{b}} V_{b}(t)+i \frac{\omega_{b}}{\Delta_{b}} \frac{\dot{V}_{b}(t)}{\Delta_{b}} \\
& +i e^{-i \Delta_{b} t} \omega_{b} \int^{t} \frac{\ddot{V}_{b}(\tau)}{\Delta_{b}^{2}} e^{i \Delta_{b} \tau} d \tau=\ldots
\end{aligned}
$$

where we dropped $\gamma_{b}$ to simplify the notation. Note that after the $n$th iteration of integration by parts, we have a correction that depends only on the instantaneous value $\propto V_{b}^{(n-1)} / \Delta_{b}^{n-1}$ and an integrand $\propto V_{b}^{(n)} / \Delta_{b}^{n} \sim\left(\Omega_{\text {peri }} / \Delta_{b}\right)^{n} V_{b}$, where $V_{b}^{(n)}$ is the $n$th time derivative of $V_{b}$. Since $\left|\Delta_{b}\right| \gg \Omega_{\text {peri }}$, the historydependent term gets progressively smaller with increasing $n$, and the instantaneous corrections to Equation (29) form a converging series (in fact, the corrections are nonzero only around a pericenter passage). This is analogous to the fact that the linear tide can be well approximated by its instantaneous equilibrium component when the tidal forcing frequency is much smaller than the mode frequency.

For the most resonant daughter mode $b$ (i.e., the $l_{b}=-m_{b}=4, \quad \omega_{b}<0 \quad f$-mode), it is possible to have $\left|\Delta_{b}\right|<\Omega_{\text {peri. }}$ In this case, the series expansion formed by integration by parts does not converge. Instead, we need to directly solve Equation (A9). To do so, we consider the following simple model of $V_{b}$ near the $k$ th pericenter passage (corresponding to time $t=t_{k}$ ):

$$
V_{b}(t)= \begin{cases}-\Delta V_{b k}, & \text { if } t<t_{k}-\frac{\pi}{\eta \Omega_{\mathrm{p}}}, \\ \Delta V_{b k} \sin \left[\eta \Omega_{\mathrm{p}}\left(t-t_{k}\right)\right], & \text { if } t_{k}-\frac{\pi}{\eta \Omega_{\mathrm{p}}} \leqslant t \leqslant t_{k}+\frac{\pi}{\eta \Omega_{\mathrm{p}}}, \\ \Delta V_{b k}, & \text { if } t>t_{k}+\frac{\pi}{\eta \Omega_{\mathrm{p}}},\end{cases}
$$

where $\eta \sim 1$ is a correction on the characteristic timescale over which $q_{a}$ changes, and we rewrote $\Omega_{\text {peri }}$ as $\Omega_{\mathrm{p}}$ in order to reduce notational clutter. With this simple model we can easily evaluate the integration around $t_{k}$ as

$$
\begin{aligned}
& \int_{t_{k}-\pi / \eta \Omega_{\mathrm{p}}}^{t_{k}+\pi / \eta \Omega_{\mathrm{p}}} \dot{V}_{b}(\tau) e^{\left(i \Delta_{b}+\gamma_{b}\right) \tau} d \tau \\
& \simeq\left[\frac{\eta \Omega_{\mathrm{p}}}{\left(\Delta_{b}+\eta \Omega_{\mathrm{p}}-i \gamma_{b}\right)} \sin \left(\frac{\Delta_{b}+\eta \Omega_{\mathrm{p}}}{\eta \Omega_{\mathrm{p}}} \pi\right)\right. \\
& \left.+\frac{\eta \Omega_{\mathrm{p}}}{\left(\Delta_{b}-\eta \Omega_{\mathrm{p}}-i \gamma_{b}\right)} \sin \left(\frac{\Delta_{b}-\eta \Omega_{\mathrm{p}}}{\eta \Omega_{\mathrm{p}}} \pi\right)\right] \Delta V_{b k},
\end{aligned}
$$

where we ignored dissipation over a time $2 \pi / \eta \Omega_{\mathrm{p}}$. We can now write mode $b$ 's amplitude as ${ }^{21}$

$$
\begin{aligned}
c_{b}(t) \simeq & \frac{\omega_{b}}{\Delta_{b}-i \gamma_{b}}\left\{V_{b}(t)+e^{-\left(i \Delta_{b}+\gamma_{b}\right) t}\right. \\
& \left.\times\left[\frac{\eta \Omega_{\mathrm{p}} \sin \left(\frac{\Delta_{b} \pm \eta \Omega_{\mathrm{p}}}{\eta \Omega_{\mathrm{p}}} \pi\right)}{\left(\Delta_{b} \pm \eta \Omega_{\mathrm{p}}-i \gamma_{b}\right)}\right] \sum_{k}^{t_{k}<t} \Delta V_{b k}\right\} .
\end{aligned}
$$

Therefore, in addition to the instantaneous term $V_{b}(t)$, there in principle should also be a history-dependent term $\sum \Delta V_{b k}$. Physically, this case can be understood by the following. As the parent mode's amplitude changes at each pericenter passage over a timescale $1 / \eta \Omega_{\text {peri }}$, its frequency content is broadened from a single delta function at $\omega_{a}$ to a band covering $\omega_{a} \pm \eta \Omega_{\text {peri }} / 2$. Since the parent-daughter detuning is small, $\left|\Delta_{b}\right|<\Omega_{\text {peri }}$, the broadened drive from the parent can now resonantly excite the daughter. This thus gives the daughter a "dynamical" component that depends on the past history $\left(\sum \Delta V_{b k}\right)$. By contrast, when the daughter is not resonant, the instantaneous "equilibrium" component dominates.

If $\sum \Delta V_{b k}$ grows diffusively (since $\Delta V_{b k} \propto q_{a}^{*} \Delta q_{a, 1}$, and the phase of $q_{a}^{*}$ can be random), then both $V_{b}$ and $\sum \Delta V_{b k}$ grow with the number of pericenter passages $k$ as $\propto k$. Therefore, the history term due to the kick on $q_{a}$, unlike the one due to $U_{a}$, can be potentially important. For simplicity, we drop it in the analysis of this paper and defer its consideration to future work.

\section{ORCID iDs}

Hang Yu (iD https://orcid.org/0000-0002-6011-6190

Nevin N. Weinberg (i) https://orcid.org/0000-0001-9194-2084

Phil Arras (iD https://orcid.org/0000-0001-5611-1349

\section{References}

Arras, P., \& Socrates, A. 2009, arXiv:0901.0735

Barker, A. J. 2011, MNRAS, 414, 1365

Burkart, J., Quataert, E., Arras, P., \& Weinberg, N. N. 2013, MNRAS, 433,332

Chatterjee, S., Ford, E. B., Matsumura, S., \& Rasio, F. A. 2008, ApJ, 686, 580 Dawson, R. I., \& Johnson, J. A. 2018, ARA\&A, 56, 175

Dawson, R. I., Murray-Clay, R. A., \& Johnson, J. A. 2015, ApJ, 798, 66 Essick, R., \& Weinberg, N. N. 2016, ApJ, 816, 18

\footnotetext{
21 In fact, this solution applies to the case where $\Omega_{\text {peri }}<\left|\Delta_{b}\right|$ as well. It is just less apparent to see why in the large- $\Delta_{b}$ case the amplitude of mode $b$ is independent of its history from Equation (A13) than from the series expansion formed by consecutive integration by part.
} 
Goldreich, P., \& Nicholson, P. D. 1977, Icar, 30, 301

Hamer, J. H., \& Schlaufman, K. C. 2019, AJ, 158, 190

Hamers, A. S., Antonini, F., Lithwick, Y., Perets, H. B., \& Portegies Zwart, S. F. 2017, MNRAS, 464, 688

Hut, P. 1981, A\&A, 99, 126

Ivanov, P. B., \& Papaloizou, J. C. B. 2004, MNRAS, 347, 437

Jermyn, A. S., Tout, C. A., \& Ogilvie, G. I. 2017, MNRAS, 469, 1768

Kochanek, C. S. 1992, ApJ, 385, 604

Kumar, P., Goldreich, P., \& Kerswell, R. 1994, ApJ, 427, 483

Kumar, P., \& Goodman, J. 1996, ApJ, 466, 946

Lai, D. 1997, ApJ, 490, 847

Lainey, V., Arlot, J.-E., Karatekin, Ö., \& van Hoolst, T. 2009, Natur, 459, 957

Landau, L. D., \& Lifshitz, E. M. 1976, Mechanics: Volume 1, Vol. 1 (Oxford: Butterworth-Heinemann)

Mankovich, C., \& Fuller, J. 2021, arXiv:2104.13385

Mardling, R. A. 1995, ApJ, 450, 722

Mayor, M., \& Queloz, D. 1995, Natur, 378, 355

Nagasawa, M., Ida, S., \& Bessho, T. 2008, ApJ, 678, 498

Ogilvie, G. I., \& Lin, D. N. C. 2004, ApJ, 610, 477

Paxton, B., Bildsten, L., Dotter, A., et al. 2011, ApJS, 192, 3
Paxton, B., Cantiello, M., Arras, P., et al. 2013, ApJS, 208, 4

Paxton, B., Marchant, P., Schwab, J., et al. 2015, ApJS, 220, 15

Paxton, B., Schwab, J., Bauer, E. B., et al. 2018, ApJS, 234, 34

Paxton, B., Smolec, R., Schwab, J., et al. 2019, ApJS, 243, 10

Press, W. H., \& Teukolsky, S. A. 1977, ApJ, 213, 183

Rasio, F. A., \& Ford, E. B. 1996, Sci, 274, 954

Schenk, A. K., Arras, P., Flanagan, É. É., Teukolsky, S. A., \& Wasserman, I. 2002, PhRvD, 65, 024001

Socrates, A., Katz, B., Dong, S., \& Tremaine, S. 2012, ApJ, 750, 106

Teyssandier, J., Lai, D., \& Vick, M. 2019, MNRAS, 486, 2265

Townsend, R. H. D., Goldstein, J., \& Zweibel, E. G. 2018, MNRAS, 475, 879

Townsend, R. H. D., \& Teitler, S. A. 2013, MNRAS, 435, 3406

Vick, M., \& Lai, D. 2018, MNRAS, 476, 482

Vick, M., \& Lai, D. 2019, PhRvD, 100, 063001

Vick, M., Lai, D., \& Anderson, K. R. 2019, MNRAS, 484, 5645

Weinberg, N. N., Arras, P., Quataert, E., \& Burkart, J. 2012, ApJ, 751, 136

Wu, Y. 2018, AJ, 155, 118

Wu, Y., \& Lithwick, Y. 2011, ApJ, 735, 109

Wu, Y., \& Murray, N. 2003, ApJ, 589, 605

Yu, H., Weinberg, N. N., \& Fuller, J. 2020, MNRAS, 496, 5482 